\title{
Evaluating Spatial and Temporal Variation in Tuzaklı Pond Water Using Multivariate Statistical Analysis
}

\author{
Arzu Aydin Uncumusaoğlu ${ }^{1 *}$, Ekrem Mutlu \\ ${ }^{1}$ Giresun University, Faculty of Engineering, Department of Environmental Engineering, Giresun, Turkey \\ ${ }^{2}$ Kastamonu University, Faculty of Fisheries, Departments of Aquaculture, Kastamonu, Turkey
}

Received: 30 August 2018

Accepted: 21 October 2018

\begin{abstract}
This study used multivariate statistical techniques to demonstrate the spatial and temporal changes in water quality, main pollutant sources and water quality classes in Tuzakl1 Pond. The water quality datasets are obtained on a monthly basis (November 2014-October 2015) using the results of 28 parameters that are obtained from three stations in the pond. Datasets are spatially and temporally assessed using statistical techniques, including one-way analysis of variance (ANOVA), Pearson's correlation, hierarchical agglomerative cluster analysis (HCA) and principal component analysis (PCA). PCA indicates the four main components responsible for the data structure, accounting for $88.31 \%$ of the total variance of the dataset. These main components are physical parameters, soluble salts (natural), ammonium and phosphorus (agricultural activity), which are nutrient elements. Furthermore, it can be temporally concluded using HCA that the summer and autumn seasons exhibit more similar characteristics as compared to those exhibited by the remaining seasons. According to the water quality and class criteria of Turkey Surface Water Management Regulation and the World Health Organisation (WHO), while this pond generally represents Class I, we observed $\mathrm{PO}_{4}^{3-}, \mathrm{SO}_{3}{ }^{2-}, \mathrm{NO}_{2}^{-}$and $\mathrm{NO}_{3}^{-}(\mathrm{Class}$ II), which resulted in slightly contaminated water.
\end{abstract}

Keywords: water quality; principal component analysis (PCA); hierarchical clustering analysis (HCA); temporal-spatial variations; Pearson correlation

\section{Introduction}

Water is the basic requirement for all life forms. Ensuring safe access to healthy water is necessary to maintain life. We can observe that the total freshwater source from all existing ecosystems is only $2.5 \%$.

*e-mail: arzu.a.uncumusaoglu@gmail.com
Furthermore, fresh water is not readily available for the utilisation of living beings because more than $68 \%$ of freshwater is located in the poles and on mountains in the form of snow and ice, which makes it more difficult to obtain. $31.4 \%$ of fresh water is present as groundwater, whereas only $0.3 \%$ of fresh water is present in surface waters. Furthermore, in the case of surface waters, the distribution of fresh water is as follows: $87 \%$ in lakes, $11 \%$ in marshes and $2 \%$ in rivers [1]. Currently, the 
world population has exceeded 7.6 billion; because the population is increasing daily, the requirement of fresh water for drinking and other activities is exhibiting a proportional increase. However, the water used by humans is contaminated with industrial wastes, sewage and wastewater, chemical fertilizers, agricultural chemicals (pesticides), radioactive wastes, animal wastes, leaks from storage areas, wastes produced as a result of global climate change, mining activities, oil tanker accidents, fossil fuel consumption and overurbanisation, as well as various other wastes. These polluted waters mix with the groundwater and the drinking water because of precipitation. They do not get sufficiently cleaned during the natural cycle and begin to pose a significant danger to living creatures.

When these pollutants are mixed with irrigation waters, significant environmental problems are observed in aquatic ecosystems (pollution, toxicity, etc.) [2-5]. The properties of water sources should be thoroughly investigated before they are directly and indirectly used. These investigations should be periodically conducted based on physical, chemical and biological examinations of water sources [6]. In addition to providing adequate safe water, the sustainable management of the water quality of surface waters using a reliable and representative quality monitoring program is one of the major tasks of the municipalities and the relevant official agencies of the state.

Lakes contain some of the most important freshwater reserves. Dam reservoirs and ponds can be used for various purposes, such as to provide drinking water, water for irrigation, utility water and to ensure energy conservation and flood prevention. Ponds are generally considered to be stagnant water bodies that are collected behind the levies built on rivers. Shallow lakes or ponds are more productive and usable in comparison [7]. They should be continuously monitored because they exhibit their own unique changes that make them special [8].

By performing various monitoring studies, multivariate statistical and computational methods - such as one-way analysis of variance (ANOVA), Pearson's correlation, hierarchical agglomerative cluster analysis (HCA), principal component analysis (PCA), factor analysis (FA) and discriminant analysis (DA) - have been developed because of their capabilities to process large amount of spatial and temporal data to understand the water quality and ecological status in a better way, to filter large datasets into meaningful ranges, to obtain useful information, to identify the relationships between relevant data and to evaluate the results [9-12].

These analyses and techniques increase the ease and clarity of the data interpretation; they can also be used to identify the probable factors that affect water systems, to identify pollution sources and to classify them using clustering based on whether the monitoring stations exhibit similar characteristics and to provide valuable tools to rapidly solve the pollution problems to ensure reliable management of water resources [13-15].
Additionally, these techniques provide reliable results while evaluating the water recycling strategies, while assesing the risk in waste water management and while assessing the groundwater hydrology and chemistry [16$18]$.

This study was consucted in Tuzaklı Pond, which is located within the borders of the Araç District of the Kastamonu Province, by obtaining the results of a total of 28 parameters between November 2014 and October 2015 using the surface water samples that have been obtained from the three stations on a monthly basis. This study intends to determine the water quality classes of the pond according to the World Health Organisation (WHO) and Turkey's Regulation on the Management of Surface Water Quality as well as the water quality classes of the inland surface water sources; in addition, this study aims to use multivariate statistical techniques to reveal both the temporal and spatial similarities or differences between the sampling stations using the wide data matrix that is obtained while evaluating the results.

\section{Material and Methods}

\section{Sample Location and Sampling}

Tuzakl1 Pond, which is located within the borders of the Araç District of Kastamonu Province, is built $6 \mathrm{~km}$ to the northeast of the district centre for irrigation purposes. The water source of the pond is Gavur Creek, along with snow and rain waters. The volume of the pond is $0.14 \mathrm{hm}^{3}$. The storage volume of the dam is $1.1 \mathrm{hm}^{3}$ and the average depth is $10.6 \mathrm{~m}$. A warm and temperate climate is observed to be dominant in the research area, and significant precipitation can be observed in the district. The annual average temperature of the region is $10.9^{\circ} \mathrm{C}$, and the average annual rainfall is $570 \mathrm{~mm}$. The hottest month of the year is July $\left(20.6^{\circ} \mathrm{C}\right)$, whereas January has the lowest average temperature at $0.5^{\circ} \mathrm{C}[19]$.

\section{Monitoring Sites}

Water samples were obtained from three designated stations (Fig. 1 and Table 1) in the pond. Sampling was conducted once a month for a year (May 2015-April 2016) in order to determine the spatial and temporal changes in water quality. The water samples were obtained by agitating 3-litre water bottles with pond

Table 1. Geographical coordinates of sampling sites.

\begin{tabular}{|c|c|c|}
\hline Sampling sites & Latitude & Longitude \\
\hline S1 & $41^{\circ} 18^{\prime} 15.24{ }^{\prime \prime} \mathrm{N}$ & $33^{\circ} 23^{\prime} 40.89^{\prime} \mathrm{E}$ \\
\hline S2 & $41^{\circ} 18^{\prime} 13.566^{\prime} \mathrm{N}$ & $33^{\circ} 23^{\prime} 29.844^{\prime} \mathrm{E}$ \\
\hline S3 & $41^{\circ} 18^{\prime} 24.40^{\prime \prime} \mathrm{N}$ & $33^{\circ} 23^{\prime} 40.58^{\prime} \mathrm{E}$ \\
\hline
\end{tabular}




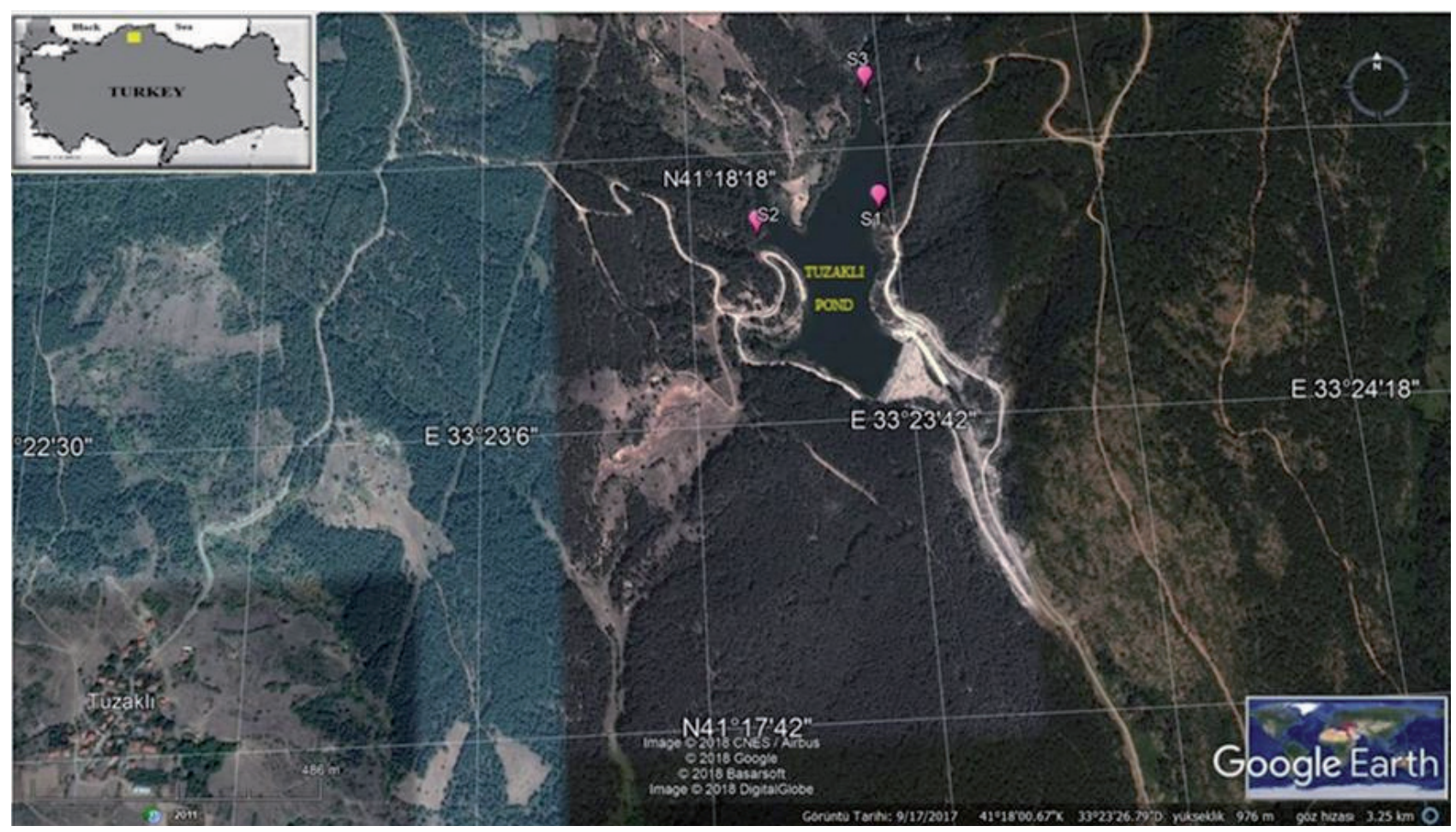

Fig. 1. Map of the study area with the locations of the sampling sites (adapted from Google Earth).

water and submerging them to a depth of $15 \mathrm{~cm}$ from the water surface.

\section{Determining Physico-Chemical Parameters}

The surface water samples were obtained from the stations that were identified in the study area. Physical and chemical analyses were conducted, and the obtained data were seasonally evaluated. The physical parameters of water quality, such as dissolved oxygen (DO), electrical conductivity (EC), salinity and water temperature (WT), were measured using the multiparameter YSI 556 MPS model device in the field. The biological oxygen demand $\left(\mathrm{BOD}_{5}\right)$, chemical oxygen demand (COD), total alkalinity (TA), total hardness (TH), nitrite nitrogen $\left(\mathrm{NO}_{2}^{-}-\mathrm{N}\right)$, nitrate nitrogen $\left(\mathrm{NO}_{3}^{-}-\mathrm{N}\right)$, ammonium nitrogen $\left(\mathrm{NH}_{4}^{+}-\mathrm{N}\right)$, sulphite $\left(\mathrm{SO}_{3}{ }^{2-}\right)$, sulphate $\left(\mathrm{SO}_{4}{ }^{2-}\right)$, potassium $(\mathrm{K}+)$, chloride $\left(\mathrm{Cl}^{-}\right)$ and phosphate-phosphorus $\left(\mathrm{PO}_{4}^{3-} \mathrm{P}\right)$ were analysed in a laboratory by employing the standard method using a spectrophotometer [20-21]. Titration was performed using sulphuric acid for total alkalinity with EDTA for total hardness, and the results were stated in terms of $\mathrm{CaCO}_{3} \mathrm{mg} \mathrm{L}^{-1}$. Whatman membrane filters were used to perform the suspended solid matter (SS) in water analysis. Water was passed through the filter paper and was further maintained for 24 hours at $103^{\circ} \mathrm{C}$, and the weight difference was calculated [1]. The amounts of $\mathrm{Ca}^{2+}, \mathrm{Mg}^{2+}$ and $\mathrm{Na}^{+}$were measured using a direct flame photometer.

Using an ICP-MS instrument, $\mathrm{Ni}^{2+}, \mathrm{Pb}^{2+}, \mathrm{Cd}^{2+}, \mathrm{Zn}^{2+}$, $\mathrm{Cu}^{2+}$ and $\mathrm{Fe}^{2+}$ heavy metal analyses were conducted based on the water samples. A calibration curve was developed using the certified multi-element standard [22].

To determine water quality classes, quality criteria assessments were conducted based on the general chemical and physico-chemical parameters according to the classes of surface waters based primarily on WHO and Tukey's Surface Water Quality Regulation of Inland Surface Water Sources [23-24].

\section{Data Treatment and Multivariate Statistical Analysis}

Using the multivariate statistical methods, it becomes easy to understand and interpret an extensive variety of data. Therefore, ANOVA, Pearson's correlation, HCA and PCA/FA were conducted. ANOVA was performed to investigate whether there was any difference between the measurements of the same observations at different times or situations with respect to any variable [25]. In this study, ANOVA was performed using Tukey's multiple-range tests that estimate whether there is any significant difference between the mean values of stations and seasons.

The correlation coefficients provide information about the strength/degree of the relationship between two variables [26]. Because of the non-uniform distribution of the measured water quality parameters, the correlation between variables can be calculated by the non-parametric Pearson's correlation coefficient (r) [27]. Furthermore, the correlation coefficient ranges from -1 to 1 and measures the degree of linear 
Table 2. Station mean values, standard deviations and ranges (min.-max.) of water quality parameters.

\begin{tabular}{|c|c|c|c|c|}
\hline & S1 & S2 & S3 & Sig. \\
\hline $\mathrm{DO}\left(\mathrm{mg} \mathrm{L}^{-1}\right)$ & $\begin{array}{c}11.705 \pm 0.9640 \\
10.28-13.17\end{array}$ & $\begin{array}{c}11.7575 \pm 0.8873 \\
10.26-13.16\end{array}$ & $\begin{array}{c}11.8033 \pm 0.8969 \\
10.30-13.20\end{array}$ & 0.966 \\
\hline Salinity (\%o) & $\begin{array}{c}0.0642 \pm 0.0303 \\
0.03-0.12\end{array}$ & $\begin{array}{c}0.0641 \pm 0.0320 \\
0.02-0.12\end{array}$ & $\begin{array}{c}0.0566 \pm 0.0287 \\
0.02-0.11\end{array}$ & 0.785 \\
\hline $\mathrm{pH}$ & $\begin{array}{c}8.138 \pm 0.1614 \\
7.91-8.41\end{array}$ & $\begin{array}{c}8.0625 \pm 0.3669 \\
7.00-8.42\end{array}$ & $\begin{array}{c}8.1225 \pm 0.1600 \\
7.90-8.39\end{array}$ & 0.736 \\
\hline WT $\left(\mathrm{C}^{\circ}\right)$ & $\begin{array}{c}12.1833 \pm 7.3180 \\
3.10-25.40\end{array}$ & $\begin{array}{c}12.3166 \pm 7.2933 \\
3.20-25.40\end{array}$ & $\begin{array}{c}12.1250 \pm 7.3039 \\
3.10-25.20\end{array}$ & 0.998 \\
\hline $\mathrm{EC}\left(\mu \mathrm{S} \mathrm{cm}^{-1}\right)$ & $\begin{array}{c}190.84 \pm 53.7855 \\
117.40-272.30 \\
\end{array}$ & $\begin{array}{c}192.3433 \pm 54.0282 \\
118.96-272.92 \\
\end{array}$ & $\begin{array}{c}189.3258 \pm 53.4892 \\
116.54-269.98\end{array}$ & 0.991 \\
\hline $\mathrm{SS}\left(\mathrm{mg} \mathrm{L}^{-1}\right)$ & $\begin{array}{c}5.7617 \pm 2.9274 \\
2.36-10.72\end{array}$ & $\begin{array}{c}5.8083 \pm 2.9383 \\
2.40-10.78\end{array}$ & $\begin{array}{c}5.7667 \pm 2.9245 \\
2.38-10.76\end{array}$ & 0.999 \\
\hline $\operatorname{COD}\left(\mathrm{mg} \mathrm{L}^{-1}\right)$ & $\begin{array}{c}3.3375 \pm 1.5024 \\
1.00-6.20\end{array}$ & $\begin{array}{c}3.4041 \pm 1.5147 \\
1.02-6.26\end{array}$ & $\begin{array}{c}3.4600 \pm 1.5705 \\
1.02-6.26\end{array}$ & 0.981 \\
\hline $\mathrm{BOD}_{5}\left(\mathrm{mg} \mathrm{L}^{-1}\right)$ & $\begin{array}{c}1.4033 \pm 0.5565 \\
0.62-2.08\end{array}$ & $\begin{array}{c}1.4733 \pm 0.5704 \\
0.66-2.20\end{array}$ & $\begin{array}{c}1.4483 \pm 0.5695 \\
0.64-2.16\end{array}$ & 0.954 \\
\hline $\mathrm{Cl}^{-}\left(\mathrm{mg} \mathrm{L}^{-1}\right)$ & $\begin{array}{c}5.365 \pm 1.1886 \\
3.38-6.64\end{array}$ & $\begin{array}{c}5.4383 \pm 1.1958 \\
3.42-6.72\end{array}$ & $\begin{array}{c}5.4033 \pm 1.2056 \\
3.36-6.70\end{array}$ & 0.989 \\
\hline $\mathrm{PO}_{4}^{3-}\left(\mathrm{mg} \mathrm{L}^{-1}\right)$ & $\begin{array}{c}0.242 \pm 0.1670 \\
0.07-0.58\end{array}$ & $\begin{array}{c}0.2518 \pm 0.1718 \\
0.06-0.59\end{array}$ & $\begin{array}{c}0.2472 \pm 0.1796 \\
0.00-0.58\end{array}$ & 0.990 \\
\hline $\mathrm{SO}_{4}^{2-}\left(\mathrm{mg} \mathrm{L}^{-1}\right)$ & $\begin{array}{c}63.54 \pm 9.5765 \\
48.78-82.26\end{array}$ & $\begin{array}{c}64.3366 \pm 9.7428 \\
48.88-82.26\end{array}$ & $\begin{array}{c}63.4817 \pm 9.5284 \\
48.84-81.04\end{array}$ & 0.971 \\
\hline $\mathrm{SO}_{3}{ }^{2-}\left(\mathrm{mg} \mathrm{L}^{-1}\right)$ & $\begin{array}{c}1.2700 \pm 0.3656 \\
0.64-1.94\end{array}$ & $\begin{array}{c}1.3416 \pm 0.3817 \\
0.72-2.06\end{array}$ & $\begin{array}{c}1.2517 \pm 0.4055 \\
0.68-1.92\end{array}$ & 0.833 \\
\hline $\mathrm{Na}^{+}\left(\mathrm{mg} \mathrm{L}^{-1}\right)$ & $\begin{array}{c}49.9017 \pm 13.0903 \\
38.62-75.30\end{array}$ & $\begin{array}{c}50.4283 \pm 13.4513 \\
38.70-76.10\end{array}$ & $\begin{array}{c}50.3983 \pm 13.1111 \\
40.12-75.90\end{array}$ & 0.994 \\
\hline $\mathrm{K}^{+}\left(\mathrm{mg} \mathrm{L}^{-1}\right)$ & $\begin{array}{c}7.2600 \pm 3.1562 \\
4.74-15.88\end{array}$ & $\begin{array}{c}7.4483 \pm 3.4777 \\
4.80-17.20\end{array}$ & $\begin{array}{c}7.4016 \pm 3.4637 \\
4.76-17.14\end{array}$ & 0.990 \\
\hline $\mathrm{TH}\left(\mathrm{CaCO}_{3} \mathrm{mg} \mathrm{L}^{-1}\right)$ & $\begin{array}{c}274.6283 \pm 39.2025 \\
241.06-381.36\end{array}$ & $\begin{array}{c}271.0250 \pm 21.8907 \\
241.48-301.92 \\
\end{array}$ & $\begin{array}{c}277.4583 \pm 42.6217 \\
239.74-397.00\end{array}$ & 0.907 \\
\hline $\mathrm{TA}\left(\mathrm{CaCO}_{3} \mathrm{mg} \mathrm{L}^{-1}\right)$ & $\begin{array}{c}273.9253 \pm 22.0545 \\
243.84-305.06\end{array}$ & $\begin{array}{c}274.8750 \pm 22.0214 \\
244.64-305.50\end{array}$ & $\begin{array}{c}272.2367 \pm 21.3965 \\
242.96-304.30\end{array}$ & 0.956 \\
\hline $\mathrm{Mg}^{2+}\left(\mathrm{mg} \mathrm{L}^{-1}\right)$ & $\begin{array}{c}39.2467 \pm 10.8953 \\
24.24-54.22\end{array}$ & $\begin{array}{c}39.2641 \pm 11.3102 \\
22.20-55.28\end{array}$ & $\begin{array}{c}39.2233 \pm 11.2971 \\
22.20-55.20\end{array}$ & 1.000 \\
\hline $\mathrm{Ca}^{2+}\left(\mathrm{mg} \mathrm{L}^{-1}\right)$ & $\begin{array}{c}46.6267 \pm 15.6070 \\
22.82-79.82\end{array}$ & $\begin{array}{c}47.0666 \pm 15.6761 \\
23.06-80.58\end{array}$ & $\begin{array}{c}46.8300 \pm 15.6997 \\
22.96-80.50\end{array}$ & 0.998 \\
\hline $\mathrm{NO}_{2}^{-}\left(\mathrm{mg} \mathrm{L}^{-1}\right)$ & $\begin{array}{l}0.0002 \pm 0.0001 \\
0.0001-0.0006\end{array}$ & $\begin{array}{c}0.0015 \pm 0.0043 \\
0.0001-0.0151\end{array}$ & $\begin{array}{l}0.0002 \pm 0.0002 \\
0.0001-0.0007\end{array}$ & 0.338 \\
\hline $\mathrm{NO}_{3}^{-}\left(\mathrm{mg} \mathrm{L}^{-1}\right)$ & $\begin{array}{c}5.092 \pm 3.113 \\
1.67-12.92\end{array}$ & $\begin{array}{c}5.272 \pm 3.3342 \\
1.68-13.10\end{array}$ & $\begin{array}{c}4.883 \pm 3.283 \\
1.64-13.06\end{array}$ & 0.958 \\
\hline $\mathrm{NH}_{4}^{+}\left(\mathrm{mg} \mathrm{L}^{-1}\right)$ & $\begin{array}{c}0.0004 \pm 0.0007 \\
0.00-0.0026\end{array}$ & $\begin{array}{c}0.0006 \pm 0.0009 \\
0.00-0.0034\end{array}$ & $\begin{array}{c}0.0006 \pm 0.0008 \\
0.00-0.0030\end{array}$ & 0.855 \\
\hline $\mathrm{Fe}^{2+}\left(\mathrm{mg} \mathrm{L}^{-1}\right)$ & $\begin{array}{c}0.0014 \pm 0.0017 \\
0.00-0.005\end{array}$ & $\begin{array}{c}0.0019 \pm 0.0022 \\
0.00-0.007\end{array}$ & $\begin{array}{c}0.0015 \pm 0.0020 \\
0.00-0.006\end{array}$ & 0.782 \\
\hline $\mathrm{Pb}^{2+}\left(\mu \mathrm{g} \mathrm{L}^{-1}\right)$ & $\begin{array}{c}0.8917 \pm 0.4231 \\
0.30-1.80\end{array}$ & $\begin{array}{c}1.0667 \pm 0.4960 \\
0.30-2.10\end{array}$ & $\begin{array}{c}0.9833 \pm 0.4821 \\
0.30-2.00\end{array}$ & 0.661 \\
\hline $\mathrm{Cu}^{2+}\left(\mu \mathrm{g} \mathrm{L}^{-1}\right)$ & $\begin{array}{c}4.0833 \pm 3.4499 \\
0.00-11.00\end{array}$ & $\begin{array}{c}5.1750 \pm 4.1631 \\
0.00-14.00\end{array}$ & $\begin{array}{c}4.7500 \pm 3.9109 \\
0.00-13.00\end{array}$ & 0.784 \\
\hline $\mathrm{Cd}^{2+}\left(\mu \mathrm{g} \mathrm{L}^{-1}\right)$ & $\begin{array}{c}0.1583 \pm 0.1084 \\
0.00-0.40\end{array}$ & $\begin{array}{c}0.1917 \pm 0.1443 \\
0.00-0.50\end{array}$ & $\begin{array}{c}0.1833 \pm 0.1467 \\
0.00-0.50\end{array}$ & 0.819 \\
\hline $\mathrm{Hg}^{2+}\left(\mu \mathrm{g} \mathrm{L}^{-1}\right)$ & $\begin{array}{c}0.0011 \pm 0.0017 \\
0.00-0.005\end{array}$ & $\begin{array}{c}0.0017 \pm 0.0024 \\
0.00-.007\end{array}$ & $\begin{array}{c}0.0015 \pm 0.0022 \\
0.00-0.006\end{array}$ & 0.832 \\
\hline
\end{tabular}


Table 2. Continued.

\begin{tabular}{|c|c|c|c|c|}
\hline \multirow{2}{*}{$\mathrm{Ni}^{2+}\left(\mu \mathrm{g} \mathrm{L}^{-1}\right)$} & $1.5000 \pm 1.2432$ & $2.1667 \pm 1.6422$ & $1.7500 \pm 1.7645$ & 0.579 \\
& $0.0-4.00$ & $0.00-5.00$ & $0.00-5.00$ & $10.5833 \pm 7.0770$ \\
$\mathrm{Zn}^{2+}\left(\mu \mathrm{g} \mathrm{L}^{-1}\right)$ & $9.5000 \pm 6.9085$ & $11.0000 \pm 7.0065$ & $2.00-24.00$ & 0.864 \\
\hline
\end{tabular}

Table 3. Seasonal mean values, standard deviations and ranges (min.-max.) of water quality parameters.

\begin{tabular}{|c|c|c|c|c|c|c|c|}
\hline & Winter & Spring & Summer & Autumn & Sig & $\begin{array}{l}\text { WHO } \\
\text { limits }\end{array}$ & $\begin{array}{l}\text { SWQMR } \\
\text { (Class) }\end{array}$ \\
\hline $\mathrm{DO}\left(\mathrm{mg} \mathrm{L}^{-1}\right)$ & $\begin{array}{l}11.78 \pm 0.54^{b} \\
10.56-12.25\end{array}$ & $\begin{array}{l}12.58 \pm 0.38^{c} \\
12.29-13.20\end{array}$ & $\begin{array}{l}11.94 \pm 0.73^{b} \\
11.25-12.92\end{array}$ & $\begin{array}{l}10.66 \pm 0.46^{\mathrm{a}} \\
10.26-11.30\end{array}$ & 0.000 & & I \\
\hline Salinity $(\%)$ & $\begin{array}{l}0.04 \pm 0.01^{\mathrm{a}} \\
0.02-0.06\end{array}$ & $\begin{array}{l}0.04 \pm 0.01^{\mathrm{a}} \\
0.02-0.05\end{array}$ & $\begin{array}{c}0.07 \pm 0.02^{\mathrm{b}} \\
0.05-0.10\end{array}$ & $\begin{array}{c}0.10 \pm 0.02^{\mathrm{c}} \\
0.07-0.12\end{array}$ & 0.000 & & \\
\hline $\mathrm{pH}$ & $\begin{array}{l}7.85 \pm 0.32^{\mathrm{a}} \\
7.00-7.99\end{array}$ & $\begin{array}{l}8.09 \pm 0.04^{b} \\
8.03-8.15\end{array}$ & $\begin{array}{c}8.24 \pm 0.06^{\mathrm{b}} \\
8.14-8.30\end{array}$ & $\begin{array}{l}8.25 \pm 0.18^{b} \\
8.00-8.42\end{array}$ & 0.000 & $6.5-8.5$ & I \\
\hline $\mathrm{WT}\left(\mathrm{C}^{\circ}\right)$ & $\begin{array}{c}4.16 \pm 1.39^{\mathrm{a}} \\
3.10-6.10\end{array}$ & $\begin{array}{l}8.15 \pm 2.66^{\mathrm{a}} \\
5.10-11.60\end{array}$ & $\begin{array}{l}16.56 \pm 3.35^{\mathrm{b}} \\
12.10-19.90\end{array}$ & $\begin{array}{l}19.60 \pm 5.35^{\mathrm{b}} \\
12.90-25.40\end{array}$ & 0.000 & & I \\
\hline $\mathrm{EC}\left(\mu \mathrm{S} \mathrm{cm}{ }^{-1}\right)$ & $\begin{array}{l}155.03 \pm 16.94^{\mathrm{a}} \\
139.99-178.36\end{array}$ & $\begin{array}{l}137.67 \pm 17.10^{\mathrm{a}} \\
116.54-157.26\end{array}$ & $\begin{array}{l}219.66 \pm 35.11^{\mathrm{b}} \\
175.96-261.42\end{array}$ & $\begin{array}{l}249.01 \pm 31.45^{b} \\
205.94-272.92\end{array}$ & 0.000 & 1500.0 & I \\
\hline $\mathrm{SS}\left(\mathrm{mg} \mathrm{L}^{-1}\right)$ & $\begin{array}{c}3.29 \pm 0.88^{\mathrm{a}} \\
2.36-4.42\end{array}$ & $\begin{array}{c}3.55 \pm 0.68^{\mathrm{a}} \\
2.70-4.08\end{array}$ & $\begin{array}{l}7.63 \pm 2.37^{b} \\
4.96-10.52\end{array}$ & $\begin{array}{l}8.59 \pm 1.88^{b} \\
6.40-10.78\end{array}$ & 0.000 & & \\
\hline $\operatorname{COD}\left(\mathrm{mg} \mathrm{L}^{-1}\right)$ & $\begin{array}{l}1.65 \pm 0.49^{\mathrm{a}} \\
1.00-2.16\end{array}$ & $\begin{array}{l}3.14 \pm 1.32^{\mathrm{b}} \\
1.68-4.84\end{array}$ & $\begin{array}{l}4.88 \pm 1.07^{c} \\
3.88-6.26\end{array}$ & $\begin{array}{c}3.75 \pm 0.65^{\mathrm{bc}} \\
2.85-4.22\end{array}$ & 0.000 & 10.0 & I \\
\hline $\mathrm{BOD}_{5}\left(\mathrm{mg} \mathrm{L}^{-1}\right)$ & $\begin{array}{l}0.76 \pm 0.07^{\mathrm{a}} \\
0.66-0.86\end{array}$ & $\begin{array}{l}1.18 \pm 0.49^{b} \\
0.62-1.82\end{array}$ & $\begin{array}{l}1.96 \pm 0.14^{\mathrm{c}} \\
1.82-2.20\end{array}$ & $\begin{array}{l}1.81 \pm 0.14^{c} \\
1.60-1.96\end{array}$ & 0.000 & & I \\
\hline $\mathrm{Cl}^{-}\left(\mathrm{mg} \mathrm{L}^{-1}\right)$ & $\begin{array}{l}4.51 \pm 1.64^{\mathrm{a}} \\
3.36-6.72\end{array}$ & $\begin{array}{l}4.67 \pm 0.68^{\mathrm{a}} \\
3.84-5.42\end{array}$ & $\begin{array}{l}6.12 \pm 0.08^{b} \\
6.02-6.24\end{array}$ & $\begin{array}{l}6.25 \pm 0.08^{b} \\
6.10-6.36\end{array}$ & 0.000 & 250.0 & I \\
\hline $\mathrm{PO}_{4}^{3-}\left(\mathrm{mg} \mathrm{L}^{-1}\right)$ & $\begin{array}{l}0.21 \pm 0.22^{\mathrm{a}} \\
0.00-0.50\end{array}$ & $\begin{array}{l}0.11^{\mathrm{a}} \pm 0.04^{\mathrm{a}} \\
0.06-0.15\end{array}$ & $\begin{array}{c}0.25 \pm 0.06^{\mathrm{ab}} \\
0.19-0.36\end{array}$ & $\begin{array}{c}0.41 \pm 0.15^{\mathrm{b}} \\
0.24-0.59\end{array}$ & 0.001 & & II \\
\hline $\mathrm{SO}_{4}{ }^{2-}\left(\mathrm{mg} \mathrm{L}^{-1}\right)$ & $\begin{array}{l}56.18 \pm 5.52^{\mathrm{a}} \\
48.78-60.24\end{array}$ & $\begin{array}{l}62.24 \pm 1.76^{\mathrm{a}} \\
59.16-64.72\end{array}$ & $\begin{array}{l}76.87 \pm 4.90^{\mathrm{b}} \\
70.74-82.26\end{array}$ & $\begin{array}{l}60.21 \pm 6.89^{\mathrm{a}} \\
52.82-68.80\end{array}$ & 0.000 & 250.0 & \\
\hline $\mathrm{SO}_{3}{ }^{2-}\left(\mathrm{mg} \mathrm{L}^{-1}\right)$ & $\begin{array}{c}0.98 \pm 0.26^{\mathrm{a}} \\
0.64-1.24\end{array}$ & $\begin{array}{l}1.60 \pm 0.35^{\mathrm{b}} \\
1.20-2.06\end{array}$ & $\begin{array}{l}1.56 \pm 0.15^{\mathrm{b}} \\
1.34-1.76\end{array}$ & $\begin{array}{c}1.02 \pm 0.23^{\mathrm{a}} \\
0.80-1.34\end{array}$ & 0.000 & & II \\
\hline $\mathrm{Na}^{+}\left(\mathrm{mg} \mathrm{L}^{-1}\right)$ & $\begin{array}{l}41.85 \pm 1.17^{\mathrm{a}} \\
40.46-43.22\end{array}$ & $\begin{array}{c}56.77 \pm 11.36^{\mathrm{b}} \\
45.10-73.16\end{array}$ & $\begin{array}{c}60.94 \pm 13.28^{b} \\
45.10-76.10\end{array}$ & $\begin{array}{l}39.78 \pm 0.76^{\mathrm{a}} \\
38.62-40.74\end{array}$ & 0.000 & 200.0 & \\
\hline $\mathrm{K}^{+}\left(\mathrm{mg} \mathrm{L}^{-1}\right)$ & $\begin{array}{l}6.42 \pm 1.10^{\mathrm{a}} \\
5.02-7.66\end{array}$ & $\begin{array}{l}8.12 \pm 1.71^{\mathrm{ab}} \\
6.76-10.64\end{array}$ & $\begin{array}{c}10.11 \pm 5.00^{\mathrm{b}} \\
6.40-17.20\end{array}$ & $\begin{array}{c}4.84 \pm 0.06^{\mathrm{a}} \\
4.74-4.92\end{array}$ & 0.002 & 12.0 & \\
\hline $\mathrm{TH}\left(\mathrm{CaCO}_{3} \mathrm{mg} \mathrm{L}^{-1}\right)$ & $\begin{array}{c}244.61 \pm 3.28^{\mathrm{a}} \\
239.74-248.98\end{array}$ & $\begin{array}{l}261.51 \pm 8.88^{\mathrm{ab}} \\
253.00-275.88\end{array}$ & $\begin{array}{l}304.87 \pm 34.79^{b} \\
286.80-397.00\end{array}$ & $\begin{array}{l}287.99 \pm 38.82^{\mathrm{ab}} \\
256.74-381.36\end{array}$ & 0.000 & & \\
\hline $\mathrm{TA}\left(\mathrm{CaCO}_{3} \mathrm{mg} \mathrm{L}^{-1}\right)$ & $\begin{array}{c}248.19 \pm 3.58^{\mathrm{a}} \\
242.96-252.66\end{array}$ & $\begin{array}{c}265.20 \pm 9.23^{\mathrm{b}} \\
256.34-280.94\end{array}$ & $\begin{array}{c}296.94 \pm 4.69^{c} \\
291.00-303.36\end{array}$ & $\begin{array}{l}282.79 \pm 18.76^{d} \\
260.66-305.50\end{array}$ & 0.000 & 200 & \\
\hline $\mathrm{Mg}^{2+}\left(\mathrm{mg} \mathrm{L}^{-1}\right)$ & $\begin{array}{l}30.08 \pm 9.18^{\mathrm{a}} \\
22.20-42.26\end{array}$ & $\begin{array}{l}29.39 \pm 3.48^{\mathrm{a}} \\
26.92-35.02\end{array}$ & $\begin{array}{l}49.42 \pm 5.14^{b} \\
42.98-55.28\end{array}$ & $\begin{array}{c}47.48 \pm .51^{\mathrm{b}} \\
44.48-50.38\end{array}$ & 0.000 & 50 & \\
\hline $\mathrm{Ca}^{2+}\left(\mathrm{mg} \mathrm{L}^{-1}\right)$ & $\begin{array}{l}30.58 \pm 9.57^{\mathrm{a}} \\
22.82-43.36\end{array}$ & $\begin{array}{c}44.57 \pm 11.93^{b} \\
33.54-62.44\end{array}$ & $\begin{array}{c}62.27 \pm 13.71^{\mathrm{c}} \\
50.60-80.58\end{array}$ & $\begin{array}{l}47.98 \pm 2.55^{\mathrm{b}} \\
44.76-51.08\end{array}$ & 0.000 & 300 & \\
\hline $\mathrm{NO}_{2}^{-}\left(\mathrm{mg} \mathrm{L}^{-1}\right)$ & $\begin{array}{c}0.0002 \pm 0.0001^{\mathrm{a}} \\
0.0001-0.0004\end{array}$ & $\begin{array}{c}0.0004 \pm 0.0002^{\mathrm{a}} \\
0.0001-0.0007\end{array}$ & $\begin{array}{c}0.0002 \pm 0.0^{\mathrm{a}} \\
0.0001-0.0002\end{array}$ & $\begin{array}{l}0.0018 \pm 0.005^{\mathrm{a}} \\
0.0001-0.0151\end{array}$ & 0.462 & & II \\
\hline $\mathrm{NO}_{3}^{-}\left(\mathrm{mg} \mathrm{L}^{-1}\right)$ & $\begin{array}{c}2.70 \pm 1.51^{\mathrm{a}} \\
1.64-4.74\end{array}$ & $\begin{array}{c}5.62 \pm 2.10^{\mathrm{bc}} \\
3.64-8.72\end{array}$ & $\begin{array}{l}8.02 \pm 4.08^{c} \\
3.52-13.10\end{array}$ & $\begin{array}{c}3.68 \pm 0.88^{\mathrm{ab}} \\
2.00-4.72\end{array}$ & 0.000 & 50 & II \\
\hline $\mathrm{NH}_{4}^{+}\left(\mathrm{mg} \mathrm{L}^{-1}\right)$ & $\begin{array}{c}0.003 \pm 0.0005^{\mathrm{a}} \\
0.0-0.0009\end{array}$ & $\begin{array}{c}0.003 \pm 0.0003^{\mathrm{a}} \\
0.0-0.0009\end{array}$ & $\begin{array}{c}0.0003 \pm 0.0002^{\mathrm{a}} \\
0.0001-0.0007\end{array}$ & $\begin{array}{l}0.0012 \pm 0.001^{\mathrm{a}} \\
0.0001-0.0034\end{array}$ & 0.053 & 35 & I \\
\hline $\mathrm{Fe}^{2+}\left(\mathrm{mg} \mathrm{L}^{-1}\right)$ & $\begin{array}{c}0.003 \pm 0.0007^{\mathrm{a}} \\
0.0-0.002\end{array}$ & $\begin{array}{c}0.0 \pm 0.0 \mathrm{a} \\
0.0-0.0\end{array}$ & $\begin{array}{c}0.0017 \pm 0.0008 b \\
0.0003-0.003\end{array}$ & $\begin{array}{c}0.0042 \pm 0.001 \mathrm{c} \\
0.003-0.007\end{array}$ & 0.000 & 0.300 & I \\
\hline
\end{tabular}


Table 3. Continued.

\begin{tabular}{|c|c|c|c|c|c|c|c|}
\hline $\mathrm{Pb}^{2+}\left(\mu \mathrm{g} \mathrm{L}^{-1}\right)$ & $\begin{array}{c}0.79 \pm 0.14^{\mathrm{ab}} \\
0.60-1.00\end{array}$ & $\begin{array}{c}1.277 \pm 0.38^{\mathrm{b}} \\
0.90-1.90\end{array}$ & $\begin{array}{c}1.19 \pm 0.63^{b} \\
0.50-2.10\end{array}$ & $\begin{array}{c}0.67 \pm 0.29^{\mathrm{a}} \\
0.30-1.00\end{array}$ & 0.005 & 10 & I \\
\hline $\mathrm{Cu}^{2+}\left(\mu \mathrm{g} \mathrm{L}^{-1}\right)$ & $\begin{array}{c}1.01 \pm 1.31^{\mathrm{a}} \\
0.00-3.00\end{array}$ & $\begin{array}{l}4.50 \pm 4.07^{b} \\
0.00-10.00\end{array}$ & $\begin{array}{l}7.78 \pm 4.06^{\mathrm{b}} \\
3.00-14.00\end{array}$ & $\begin{array}{c}5.00 \pm 1.41^{\mathrm{b}} \\
3.00-7.00\end{array}$ & 0.001 & 20 & I \\
\hline $\mathrm{Cd}^{2+}\left(\mu \mathrm{g} \mathrm{L}^{-1}\right)$ & $\begin{array}{c}0.08 \pm 0.07^{\mathrm{a}} \\
0.0-0.20\end{array}$ & $\begin{array}{c}0.10 \pm 0.00^{\mathrm{a}} \\
0.10-0.10\end{array}$ & $\begin{array}{c}0.26 \pm 0.09^{b} \\
0.20-0.40\end{array}$ & $\begin{array}{c}0.28 \pm 0.16^{\mathrm{b}} \\
0.10-0.50\end{array}$ & 0.000 & & I \\
\hline $\mathrm{Hg}^{2+}\left(\mu \mathrm{g} \mathrm{L}^{-1}\right)$ & $\begin{array}{c}0.001 \pm 0.001^{\mathrm{a}} \\
0.0-0.004\end{array}$ & $\begin{array}{c}0.00 \pm 0.0^{\mathrm{a}} \\
0.0-0.00\end{array}$ & $\begin{array}{c}0.012 \pm 0.001^{\mathrm{a}} \\
0.0-0.004\end{array}$ & $\begin{array}{c}0.0035 \pm 0.002^{\mathrm{b}} \\
0.0007-0.007\end{array}$ & 0.001 & & I \\
\hline $\mathrm{Ni}^{2+}\left(\mu \mathrm{g} \mathrm{L}^{-1}\right)$ & $\begin{array}{c}1.11 \pm 1.54^{\mathrm{a}} \\
0.0-4.00\end{array}$ & $\begin{array}{c}0.63 \pm 1.06^{\mathrm{a}} \\
0.0-3.00\end{array}$ & $\begin{array}{c}1.89 \pm 0.60^{\mathrm{a}} \\
1.00-3.00\end{array}$ & $\begin{array}{c}3.56 \pm 1.13^{\mathrm{b}} \\
2.00-5.00\end{array}$ & 0.000 & & I \\
\hline $\mathrm{Zn}^{2+}\left(\mu \mathrm{g} \mathrm{L}^{-1}\right)$ & $\begin{array}{c}3.89 \pm 2.03^{\mathrm{a}} \\
1.00-7.00\end{array}$ & $\begin{array}{c}8.75 \pm 5.85^{\mathrm{ab}} \\
2.00-17.00\end{array}$ & $\begin{array}{c}17.00 \pm 6.14^{\mathrm{c}} \\
9.00-25.00\end{array}$ & $\begin{array}{c}11.00 \pm 5.32^{\mathrm{bc}} \\
5.00-18.00\end{array}$ & 0.000 & 10 & I \\
\hline
\end{tabular}

relationship between two variables. If $\mathrm{r}$ is close to -1 , a strong and negative linear relationship is observed between the two variables, whereas if $r$ is close to +1 , a strong and positive linear relationship is observed between the two variables [28].

It is assumed that the clusters that are obtained because of clustering analysis will be as homogeneous as possible within themselves and as heterogeneous as possible among themselves. HCA is a combination of techniques that can be used to classify clusters based on the similarities and differences between large datasets [29]. Clustering can be either hierarchical clustering or non-hierarchical clustering. The most extensively used method is hierarchical clustering [30]. In the hierarchical agglomerative clustering method, the distance between the samples is considered to be a measure of similarity. A dendrogram visually summarizes the groups and their proximity to these groups. HCA was used to observe the clustering of the water-quality dataset of Tuzaklı Pond. When this analysis was conducted, Ward's method was considered to serve as the similarity criterion [31].

FA is a collection of methods that are often used in situations when it is uncertain whether a large number of variables can be expressed using a few basic variables; it is also intended to discover a small number of new independent variables that are conceptually meaningful with minimum loss of information from a large number of inter-related variables, which are difficult to interpret. The Kaiser-Meyer-Olkin (KMO) and Bartlett tests were applied before conducting the PCA. In this study, the sufficiency of KMO is 0.633 . The Bartlett test $(\mathrm{P}=0)$ indicates that the variables are irrelevant. The KMO value should be greater than 0.5 ; otherwise the dataset is considered to be not suitable to conduct PCA [32].

Analytical data have been standardised based on z-scale to avoid misclassification due to the large differences between the data densities [33]. In this study, an eigenvalue greater than 1 is considered to be significant, and the factors with eigenvalues that are greater than or equal to 1 are considered to be the possible inventory sources in the data. However, the factor that exhibits the highest eigenvector sum is given the highest priority. Varimax normalisation has been used to interpret the results. The factor loads were classified corresponding to the absolute loading values of $>0.75,0.75-0.50$ and $0.50-0.30$ as 'strong,' 'medium' and 'weak,' respectively [34]. All the statistical analyses were performed using SPSS for Windows version 21.0.

\section{Results and Discussion}

The averages, standard deviations and minimummaximum values of the water quality parameters based on the stations and seasonal variations are presented in Tables 2 and 3. The water quality classifications based on the minimum and maximum values of the water quality parameters were performed according to the WHO and the Turkish Water Quality Standards and Surface Water Quality Classification Regulation (SWQMR) that was published in an official gazette dated 08.10.2016, number 29797 (Tables 2 and 3) [23-24]. Furthermore, the annual mean values and standard deviation of the water quality parameters were determined according to the analysis results: $\mathrm{DO}=11.76 \pm 0.89 \mathrm{mg} \mathrm{L}^{-1}$, salinity $=0.062 \pm 0.030 \%, \mathrm{pH}=8.11 \pm 0.244$, water temperature $=12.21 \pm 7.09^{\circ} \mathrm{C}, \mathrm{EC}=190.84 \pm 52.22 \mu \mathrm{s} \mathrm{cm}^{-1}$, $\mathrm{SS}=5.78 \pm 2.85 \mathrm{mg} \mathrm{L}{ }^{-1}, \mathrm{COD}=3.41 \pm 1.49 \mathrm{mg} \mathrm{L}^{-1}$, BOD5 $=1.44 \pm 0.55 \mathrm{mg} \mathrm{L}^{-1},\left[\mathrm{Cl}^{-}\right]=5.40 \pm 1.16 \mathrm{mg} \mathrm{L}^{-1}$, $\left[\mathrm{PO}_{4}{ }^{3-}\right]=0.25 \pm 0.17 \mathrm{mg} \mathrm{L}^{-1},\left[\mathrm{Na}^{+}\right]=50.24 \pm 12.84 \mathrm{mg} \mathrm{L}^{-1}$, $\left[\mathrm{K}^{+}\right]=7.37 \pm 3.27 \mathrm{mg} \mathrm{L}^{-1},\left[\mathrm{SO}_{4}{ }^{2-}\right]=63.79 \pm 9.35 \mathrm{mg} \mathrm{L}^{-1}$, $\left[\mathrm{SO}_{3}{ }^{2-}\right]=1.29 \pm 0.38 \mathrm{mg} \mathrm{L} \mathrm{L}^{-1}, \mathrm{TH}=274.37 \pm 34.81$ $\mathrm{CaCO}_{3} \mathrm{mg} \mathrm{L}^{-1}, \mathrm{TA}=273.68 \pm 21.22 \mathrm{CaCO}_{3} \mathrm{mg} \mathrm{L}^{-1}$, $\left[\mathrm{Ca}^{2+}\right]=46.84 \pm 15.21 \mathrm{mg} \quad \mathrm{L}^{-1}, \quad\left[\mathrm{Mg}^{2+}\right]=$ $39.24 \pm 10.85 \mathrm{mg} \mathrm{L}^{-1},\left[\mathrm{NO}_{2}^{-}\right]=0.0006 \pm 0.0025 \mathrm{mg} \mathrm{L}^{-1}$, $\left[\mathrm{NO}_{3}^{-}\right]=5.08 \pm 3.16 \mathrm{mg} \quad \mathrm{L}^{-1}, \quad\left[\mathrm{NH}_{4}^{+}\right]=$ $0.0005 \pm 0.0008 \mathrm{mg} \mathrm{L}^{-1},\left[\mathrm{Fe}^{2+}\right]=0.0016 \pm 0.0019 \mathrm{mg} \mathrm{L}^{-1}$, $\left[\mathrm{Pb}^{2+}\right]=0.9806 \pm 0.460 \mu \mathrm{g} \quad \mathrm{L}^{-1}, \quad\left[\mathrm{Hg}^{2+}\right]=$ $0.0014 \pm 0.002 \mu \mathrm{g} \mathrm{L} \mathrm{L}^{-1},\left[\mathrm{Ni}^{2+}\right]=1.806 \pm 1.546 \mu \mathrm{g} \mathrm{L}^{-1}$, $\left[\mathrm{Cu}^{2+}\right]=4.669 \pm 3.769 \mu \mathrm{g} \mathrm{L}^{-1},\left[\mathrm{Cd}^{2+}\right]=0.178 \pm 0.131 \mu \mathrm{g} \mathrm{L}^{-1}$ and $\left[\mathrm{Zn}^{2+}\right]=10.36 \pm 6.825 \mu \mathrm{g} \mathrm{L} \mathrm{L}^{-1}$. In this study, a statistically significant difference $(\mathrm{P}>0.05)$ is not observed based on the results of ANOVA, and the $\mathrm{P}$ values are presented in Table 2.

Statistically significant differences have been observed according to the results of ANOVA between 


\begin{tabular}{|c|c|c|c|c|c|c|c|c|c|c|c|c|c|c|c|c|c|c|c|c|c|c|c|c|c|c|c|c|c|c|c|}
\hline స̃ & & & & & & & & & & & & & & & & & & & & & & & & & & & & & & & - \\
\hline$i \bar{z}$ & & & & & & & & & & & & & & & & & & & & & & & & & & & & & & - & $\begin{array}{l}\text { के } \\
\text { के }\end{array}$ \\
\hline ID & & & & & & & & & & & & & & & & & & & & & & & & & & & & & & 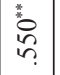 & $\begin{array}{c}\infty \\
\stackrel{\infty}{\longrightarrow}\end{array}$ \\
\hline 全 & & & & & & & & & & & & & & & & & & & & & & & & & & & & & $\begin{array}{l}4 \\
0 \\
0\end{array}$ & $\stackrel{\circ}{m}$ & $\stackrel{0}{\circ}$ \\
\hline$\exists$ & & & & & & & & & & & & & & & & & & & & & & & & & & & - & $\underset{9}{g}$ & $\underset{i}{\exists}$ & 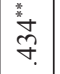 & \begin{tabular}{l}
\multirow{2}{*}{} \\
号
\end{tabular} \\
\hline$\frac{10}{2}$ & & & & & & & & & & & & & & & & & & & & & & & & & & - & 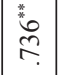 & "ू. & tot & oे & $\begin{array}{c}0 \\
0 \\
0 \\
0\end{array}$ \\
\hline $\begin{array}{l}0 \\
0 \\
0\end{array}$ & & & & & & & & & & & & & & & & & & & & & & & & & - & 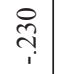 & त़ & 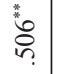 & $\stackrel{*}{*}$ & 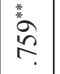 & : \\
\hline$\overline{\mathrm{Z}}^{+}$ & & & & & & & & & & & & & & & & & & & & & & & & - & $\stackrel{m}{m}$ & $\tilde{丶}_{i}$ & đ̊ & $\frac{n}{n}$ & $\vec{\Phi}$. & 莡 & $\begin{array}{l}\stackrel{*}{*} \\
\stackrel{n}{n}\end{array}$ \\
\hline$\stackrel{0}{z}$ & & & & & & & & & & & & & & & & & & & & & & - & $-z$ & $\bar{\sigma}$. & $\begin{array}{l}n \\
\stackrel{n}{0} \\
i\end{array}$ & $\begin{array}{l}x^{*} \\
\infty \\
\infty\end{array}$ & $\stackrel{*}{\circ}$ & $\tilde{\sigma}_{i}$ & $\overrightarrow{\hat{n}}$ & $\stackrel{?}{?}$ & $\begin{array}{l}* \\
\infty \\
\infty \\
\infty \\
0.0\end{array}$ \\
\hline$\stackrel{8}{z}^{2}$ & & & & & & & & & & & & & & & & & & & & & - & $\stackrel{9}{7}$ & $b^{2}$ & $\bar{\exists}$ & $\frac{\mathcal{G}}{i}$ & $\mid \begin{array}{c}0 \\
0 \\
i \\
i\end{array}$ & $\begin{array}{c}\mathbb{Z} \\
i \\
i\end{array}$ & 范 & $\vec{m}$ & $\begin{array}{l}\hat{E} \\
\text {. }\end{array}$ & $\begin{array}{c}n \\
o \\
i\end{array}$ \\
\hline తే & & & & & & & & & & & & & & & & & & & & - & $\delta$ & 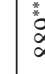 & 8 & है. & $\begin{array}{l}\infty \\
\stackrel{\sim}{\imath} \\
\end{array}$ & $\stackrel{m}{\stackrel{m}{0}}$ & $\frac{\pi}{\sigma}$ & $\begin{array}{l}\text { : } \\
m \\
m\end{array}$ & $\stackrel{0}{\circ}$ & 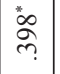 & $\begin{array}{l}0 \\
\vdots \\
\vdots \\
\infty\end{array}$ \\
\hline 管 & & & & & & & & & & & & & & & & & & & - & 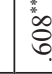 & $y$ & 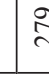 & $\frac{1}{4}$ & ָิ & $\begin{array}{l}0 \\
\infty \\
\vdots \\
b\end{array}$ & 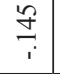 & 䒺 & 离 & 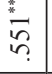 & 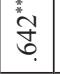 & 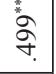 \\
\hline 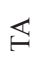 & & & & & & & & & & & & & & & & & & - & $\frac{n}{n}$ & $\mid \begin{array}{l}0 \\
\infty \\
\infty \\
0 \\
0\end{array}$ & ริ & $\widetilde{c}$ & $\hat{\imath}$ & $\stackrel{\vec{\infty}}{\underset{i}{0}}$ & o̊̀े & $\hat{\tilde{\theta}}$ & $\frac{n}{i n}$ & $\stackrel{\substack{* \\
\infty}}{\stackrel{0}{c}}$ & $\frac{n}{m}$ & $\stackrel{\sim}{ָ}$ & 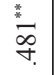 \\
\hline $\mathbb{F}$ & & & & & & & & & & & & & & & & & - & 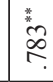 & $\frac{5}{6}$ & {$\left[\begin{array}{l}0 \\
⿱ 乛 \\
7\end{array}\right.$} & $\cong$ & $\stackrel{\infty}{\simeq}$ & $\stackrel{1}{?}$ & $\overrightarrow{\mathcal{T}}$ & તે & $\stackrel{2}{\stackrel{2}{i}}$ & 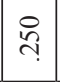 & 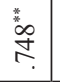 & 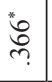 & $\stackrel{\infty}{\stackrel{\infty}{-}}$ & $\frac{\infty}{\uparrow}$ \\
\hline \pm & & & & & & & & & & & & & & & & - & Iै & స్ & $\frac{8}{1}$ & : & $\tilde{g}$ & $\underbrace{*}_{\infty}$ & 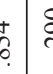 & ণે & $\begin{array}{l}\stackrel{\infty}{\infty} \\
\stackrel{1}{1}\end{array}$ & 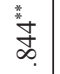 & $\begin{array}{l}* \\
\vdots \\
6\end{array}$ & $\underset{7}{\stackrel{0}{7}}$ & 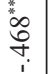 & $\underset{\vec{I}}{\vec{P}}$ & $\stackrel{*}{ \pm}$ \\
\hline$\tilde{z}$ & & & & & & & & & & & & & & & - & 辛 & $\stackrel{\circ}{\circ}$ & अे & 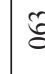 & "t. & 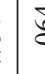 & $\circ$ & 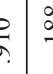 & 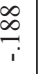 & ô & 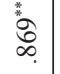 & $\mid \begin{array}{l}* \\
\infty \\
\vdots \\
\imath\end{array}$ & 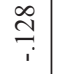 & $\begin{array}{l}\infty \\
\infty \\
\infty \\
i \\
i\end{array}$ & 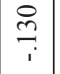 & i. \\
\hline $\begin{array}{l}\dot{x}^{-} \\
\tilde{n}^{2}\end{array}$ & & & & & & & & & & & & & & - & วิ & $\begin{array}{l}4 \\
4 \\
n \\
n\end{array}$ & $\stackrel{\overbrace{}}{\nearrow}$ & 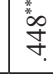 & $\begin{array}{l}\infty \\
d \\
d\end{array}$ & fo & 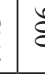 & 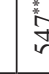 & $\begin{array}{l}f \\
t\end{array}$ & $\begin{array}{l}\stackrel{0}{\infty} \\
? \\
?\end{array}$ & 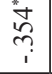 & $\stackrel{n}{\stackrel{n}{f}}$ & \begin{tabular}{|l}
0 \\
$\infty$ \\
$\infty$ \\
$\infty$ \\
+
\end{tabular} & $\exists$ & $\stackrel{\text { }}{*}$ & \begin{tabular}{l}
$\infty$ \\
$\infty$ \\
\multirow{\gamma}{*}{} \\
$i$
\end{tabular} & 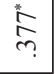 \\
\hline $\begin{array}{l}\dot{1}+ \\
0^{+}\end{array}$ & & & & & & & & & & & & & - & ôे & 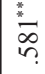 & مे & 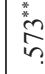 & 응 & 今ે & $\stackrel{3}{3}$ & 4 & $\int^{*}$ & 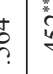 & 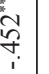 & $\stackrel{े}{े}_{i}$ & $\stackrel{\overbrace{}}{~}$ & $\mid$ & \begin{tabular}{l}
$\stackrel{*}{*}$ \\
\multirow{f}{*}{. }
\end{tabular} & $\begin{array}{c}\infty \\
\stackrel{\infty}{\rightarrow} \\
i\end{array}$ & 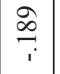 & 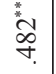 \\
\hline $0^{+}$ & & & & & & & & & & & - & & $\vec{c}$ & 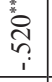 & 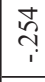 & ?. & $\bar{g}$ & $\overbrace{}^{\circ}$ & كُ & $\widehat{\widehat{\vartheta}}$ & I & $\tilde{\tilde{c}}$ & $\hat{c}_{\widehat{c}}$ & $\stackrel{0}{\circ}$ & ta & $\stackrel{i}{i}$ & \begin{tabular}{l}
0 \\
\multirow{2}{*}{}
\end{tabular} & $\stackrel{ \pm}{\Xi}$ & 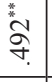 & $\begin{array}{l}0 \\
\infty \\
\infty \\
\infty \\
0\end{array}$ & 定 \\
\hline$\dot{\bar{U}}$ & & & & & & & & & & - & ? & & $\underset{\exists}{\exists}$ & $\stackrel{n}{o}_{i}$ & $\stackrel{\infty}{\stackrel{\infty}{\sim}}$ & Ĩ & $\frac{\mathrm{s}}{\mathrm{f}}$ & ڤे & ळ & 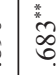 & જી & $\stackrel{*}{\forall}$ & $\frac{t}{t}$ & 炙 & $\frac{{ }^{*}}{\square}$ & 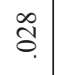 & $\mid \begin{array}{l}\tilde{n} \\
\infty \\
\infty \\
n\end{array}$ & $\stackrel{*}{\vec{\sigma}}$ & $\begin{array}{l}\tilde{n}^{2} \\
\tilde{n}\end{array}$ & 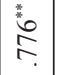 & 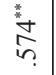 \\
\hline రి & & & & & & & & & - & 莣 & 일 & & $\tilde{b}$ & : & 点 & $\overrightarrow{\text { ले }}$ & : & $\begin{array}{l}\underbrace{\prime}_{\infty} \\
\vdots \\
\infty \\
\infty\end{array}$ & $\begin{array}{l}\vdots \\
\vdots \\
\infty \\
c\end{array}$ & $\left(\begin{array}{l}8 \\
\infty \\
\infty\end{array}\right.$ & $i$ & 变 & $?$ & $\stackrel{0}{1}$ & 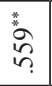 & $\begin{array}{l} \\
\text { d̦ } \\
\end{array}$ & 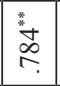 & 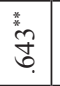 & ले & $\begin{array}{l}\vdots \\
\vdots \\
\sim \\
r\end{array}$ & 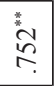 \\
\hline ชิ & & & & & & & & - & $\begin{array}{l}* \\
\infty \\
\$\end{array}$ & 然 & ฮี & & है. & $\vec{n}$ & \begin{tabular}{l}
\multirow{2}{*}{} \\
$\hat{\sigma}$
\end{tabular} & 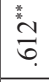 & $\stackrel{\circ}{0}$ & $\frac{*}{\infty}$ & : & 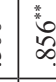 & 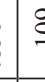 & $\underbrace{2}_{0}$ & 8 & $\exists$ & లి & \begin{tabular}{|l}
$\vdots$ \\
$\vdots$ \\
$f$ \\
$\ddots$
\end{tabular} & $\begin{array}{c}* \\
\infty \\
\infty \\
0\end{array}$ & $\stackrel{0}{0}$ & $\stackrel{\circ}{\ni}$ & 号 & $\begin{array}{l}n \\
i \\
c \\
?\end{array}$ \\
\hline$\tilde{\infty}$ & & & & & & & - & 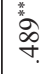 & $\stackrel{0}{n}$ & 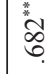 & 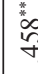 & & (t) & 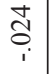 & $\stackrel{\varkappa}{\stackrel{1}{\prime}}$ & 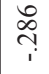 & مُ & ". & 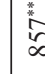 & ? & 3 & 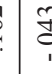 & $f_{i}^{t}$ & $\stackrel{9}{7}$ & 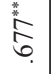 & 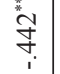 & $\stackrel{2}{\ni}$ & $\begin{array}{l}\stackrel{*}{*} \\
\infty \\
\infty\end{array}$ & $\stackrel{*}{\gtrless}$ & $\vec{n}$ & $\stackrel{\hat{\sigma}}{\circ}$ \\
\hline 㶵 & & & & & 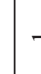 & - & $\begin{array}{l}n \\
\vdots \\
\vdots \\
o\end{array}$ & 萬 & $\frac{*}{n}$ & $\frac{\stackrel{*}{*}}{\stackrel{*}{*}}$ & in & & $\vec{c}$ & $\underset{8}{\stackrel{8}{i}}$ & ì & $\frac{0}{?}$ & 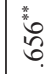 & $\stackrel{\infty}{\stackrel{\infty}{r}}$ & da & 今े & $y$ & : & $\begin{array}{l}5 \\
\\
i\end{array}$ & $\stackrel{+}{\stackrel{+}{n}}$ & $\stackrel{n}{\Omega}$ & $\mid \begin{array}{l}* \\
0 \\
⿱ 亠 䒑 \\
i\end{array}$ & $\hat{\jmath}$ & $\begin{array}{l}{ }^{*} \\
\infty \\
\infty\end{array}$ & $\underset{\substack{* \\
\infty}}{\stackrel{0}{c}}$ & $\frac{0}{0}$ & 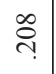 \\
\hline$\xi$ & & & & - & 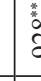 & i & $\bar{s}$ & 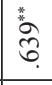 & $\begin{array}{l}4 \\
\infty \\
\infty\end{array}$ & ڤُ & f & & 昶 & $\begin{array}{l}0 \\
\text { है } \\
\end{array}$ & $\stackrel{े}{े}_{i}$ & $\frac{n}{n}$ & مُ & $\stackrel{\infty}{\infty} \underset{\infty}{\infty}$ & 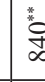 & $\frac{t}{n}$ & 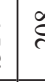 & $\stackrel{n}{=}$ & $f$ & 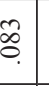 & 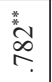 & $\begin{array}{c}\infty \\
\stackrel{\infty}{i} \\
i\end{array}$ & $\begin{array}{l}\ddot{2} \\
\ddot{m}\end{array}$ & $\stackrel{*}{\stackrel{*}{c}}$ & 落 & 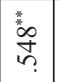 & 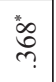 \\
\hline T & & & - & in & 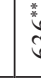 & 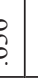 & $\begin{array}{l}0 \\
0 \\
0 \\
0 \\
0\end{array}$ & 等 & 華 & 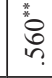 & సี & & $\begin{array}{l}8 \\
0 \\
0 \\
n\end{array}$ & $\stackrel{\infty}{-}$ & $\stackrel{\infty}{=}$ & $\overrightarrow{\hat{\theta}_{i}}$ & "艹 & 营 & ర్ & ثิ & ב & $\ddot{3}$ & Ș & of & $\begin{array}{l}* \\
\stackrel{*}{n}\end{array}$ & $\mid \begin{array}{c}0 \\
0 \\
i \\
i\end{array}$ & $\begin{array}{c}* \\
\hat{n} \\
m\end{array}$ & \begin{tabular}{l}
$m_{0}^{*}$ \\
\multirow{f}{*}{}
\end{tabular} & 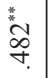 & $\stackrel{*}{\sim}$ & $\frac{\infty}{m}$ \\
\hline 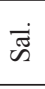 & & - & 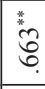 & $\stackrel{m}{m}$ & & 8 & 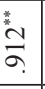 & $\begin{array}{l} \\
\end{array}$ & 莡 & 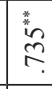 & है & & $\tilde{n}$ & $\begin{array}{l}n \\
8 \\
i \\
\end{array}$ & 尺े & 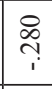 & 年 & 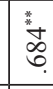 & $\frac{\infty}{\infty}$ & 亏̆ & 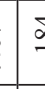 & $\bar{c}$ & ti & $\stackrel{8}{\circ}$ & $\begin{array}{l}* \\
\tilde{\omega} \\
\infty\end{array}$ & 学 & \begin{tabular}{|l|}
\multirow{j}{*}{} \\
\end{tabular} & 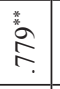 & $\begin{array}{l}* \\
\tilde{\infty} \\
\infty \\
\infty\end{array}$ & \begin{tabular}{|l|} 
\\
$\vdots$ \\
0 \\
0 \\
0
\end{tabular} & $\begin{array}{l}\infty \\
\sim \\
\end{array}$ \\
\hline ̊ & - & \begin{tabular}{l}
0 \\
\multirow{2}{*}{} \\
$i$ \\
$i$
\end{tabular} & ְె. & 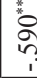 & & 6 & : & $\stackrel{9}{9}$ & $\underset{i}{\infty}$ & 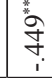 & ¿ & & ¿્૫ & 势 & $\stackrel{*}{\stackrel{*}{*}}$ & $\stackrel{*}{\stackrel{*}{r}}$ & 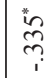 & 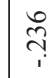 & $\mid \begin{array}{l}* \\
b \\
b \\
b \\
n\end{array}$ & $\infty$ & 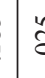 & $\tilde{n}$ & $\hat{?}$ & ثે & 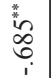 & 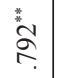 & $\frac{\infty}{m}$ & 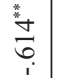 & 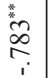 & $\begin{array}{c}0 \\
\infty \\
\infty \\
i \\
i\end{array}$ & 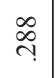 \\
\hline & 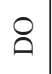 & $\dot{\vec{n}}$ & : & 5 & & & $\infty$ & ชิ & $\hat{0}^{n}$ & $\tau$ & 0 & & $0^{+}$ & $\begin{array}{l}\dot{D}^{m} \\
\overbrace{}^{m}\end{array}$ & $\ddot{z}$ & \pm & 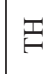 & 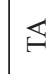 & $\sum^{+}$ & U & 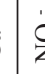 & 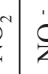 & 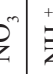 & $\mathbf{T}^{+}$ & 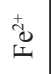 & $\begin{array}{l}+\overrightarrow{0} \\
\overrightarrow{2}\end{array}$ & $\mid \begin{array}{l} \pm \\
\Xi \\
\Xi\end{array}$ & 壱 & 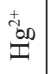 & 莣 & 䞟 \\
\hline
\end{tabular}


the mean values of the seasons $(\mathrm{P}<0.05)$, and these differences are presented in Table 3 using different letters and by specifying the "P" values.

The dissolved oxygen level of Tuzaklı Pond varied between 10.26 and $13.20 \mathrm{mg} \mathrm{L}^{-1}$. The lowest value was observed at S2 (Site 2) in September, whereas the highest value was observed at S3 (Site 3) in May. There is no apparent danger for aquatic life in terms of dissolved oxygen. According to the SWQMR and from the perspective of DO, this pond can be classified as Class I (>8 $\mathrm{mg} \mathrm{L}^{-1}$ ) (Table 3 ). According to the regulation, Class I indicates "high-quality surface water that has a high potential to be used as drinking water and that can be used for recreational purposes, including activities such as swimming that require body contact, and that can be used to breed trout or for animal husbandry and farming requirements" [23-24]. The DO at $\mathrm{P}<0.01$ and $\mathrm{P}<0.05$ significance levels exhibits a high positive significance $(\mathrm{r} \geq 0.5)$ in relation to $\mathrm{Na}^{+}, \mathrm{K}^{+}, \mathrm{SO}_{3}{ }^{2-}, \mathrm{NO}_{3}{ }^{-}$and $\mathrm{Pb}^{2+}$, and a negative significance relationship with salinity, $\mathrm{pH}$, WT, EC, SS, BOD $, \mathrm{Cl}^{-}, \mathrm{PO}_{4}^{3-}, \mathrm{TA}, \mathrm{TH}, \mathrm{Mg}^{2+} \mathrm{NH}_{4}^{+} \mathrm{Fe}^{2+}$, $\mathrm{Cd}^{2+}, \mathrm{Ni}^{2+}$ and $\mathrm{Hg}^{2+}$ (Table 4).

In this study, salinity was observed to be between 0.02 and 0.12 (\%). The lowest values were observed at S2 and S3 in January and March, whereas the highest value were at S1 and S2 in October. The salinity changes observed in the pond are suitable for maintaining aquatic life. The salinity parameter at $\mathrm{P}<0.01$ and $\mathrm{P}<0.05$ significance levels exhibits a positive significance $(r>0.5)$ relationship with $\mathrm{pH}, \mathrm{WT}, \mathrm{EC}$, $\mathrm{SS}, \mathrm{BOD}_{5}, \mathrm{Cl}^{-}, \mathrm{PO}_{4}^{3-}, \mathrm{TA}, \mathrm{TH}, \mathrm{Mg}^{2+}, \mathrm{Fe}^{2+}, \mathrm{Cd}^{2+}, \mathrm{Hg}^{2+}$ and $\mathrm{Ni}^{2+}$, whereas it exhibits a negative significance relation with $\mathrm{DO}, \mathrm{SO}_{3}{ }^{2-}, \mathrm{Na}^{+}, \mathrm{K}^{+}, \mathrm{NO}_{2}^{-}, \mathrm{NO}_{3}^{-}$and $\mathrm{Pb}^{2+}$ (Table 4).

The $\mathrm{pH}$ level of the pond varied between 7.00 and 8.42. The lowest $\mathrm{pH}$ was detected at $\mathrm{S} 2$ in January, while the highest $\mathrm{pH}$ was detected in October at the same station. The $\mathrm{pH}$ of the pond is Class I (6.5-8.5) (Table 3) [23-24]. The $\mathrm{pH}$ at $\mathrm{P}<0.01$ and $\mathrm{P}<0.05$ significance levels exhibit a positive significance $(\mathrm{r}>0.5)$ with salinity, WT, EC, SS, COD, $\mathrm{BOD}_{5}, \mathrm{Cl}^{-}$, TH, TA, $\mathrm{Cl}^{-}, \mathrm{Mg}^{2+}, \mathrm{Fe}^{2+}, \mathrm{Cd}^{2+}, \mathrm{K}^{+}, \mathrm{NO}_{2}^{-}$and $\mathrm{NH}_{4}^{+}$, and a negative significance relationship with $\mathrm{Pb}^{2+}$ (Table 4).

The water temperature varied between $3.10^{\circ} \mathrm{C}$ and $25.40^{\circ} \mathrm{C}$. The lowest temperature levels were observed at S1 and S3 in January, whereas the highest were at S1 and S2 in October. According to the inland water quality criteria of SWQMR, the water temperature class of the pond is determined to be class II $\left(>25^{\circ} \mathrm{C}\right)$ (Table 3 ) [23-24]. In the regulation, this class is defined as containing "water with low pollution; surface waters with potential of being used as drinking water; water that can be used for recreational purposes; water that can be used to breed fish other than trout and water that can be used for irrigation if the quality criteria determined by the current legislation are satisfied." This parameter at $\mathrm{P}<0.01$ and $\mathrm{P}<0.05$ significance levels exhibits a positive significance $(r>0.7)$ relationship with salinity, $\mathrm{pH}, \mathrm{EC}, \mathrm{SS}, \mathrm{COD}, \mathrm{BOD}_{5}, \mathrm{TA}, \mathrm{Mg}^{2+}, \mathrm{Fe}^{2+}$, and
$\mathrm{Cd}^{2+}$, and a negative significance relationship with DO, $\mathrm{Na}^{+}, \mathrm{K}^{+}, \mathrm{NO}_{2}^{-}$and $\mathrm{Pb}^{2+}$ (Table 4).

The EC value of the pond varied between 116.54 and $272.92 \mu \mathrm{S} \mathrm{cm}^{-1}$. The lowest level was observed at S3 in March, while the highest level was observed at $\mathrm{S} 2$ in October. According to the classification criteria of SWQMR and the WHO regulations, Tuzaklı Pond is Class $\mathrm{I}$ in terms of $\mathrm{EC}\left(<00 \mu \mathrm{S} \mathrm{cm} \mathrm{cm}^{-1}\right)$ (Table 3) [23-24]. The EC at $\mathrm{P}<0.01$ and $\mathrm{P}<0.05$ significance levels, parameters with high positive significance $(\mathrm{r} \geq 0.7)$ are salinity, WT, $\mathrm{SS}, \mathrm{BOD}_{5}, \mathrm{Cl}^{-}, \mathrm{TA}, \mathrm{Mg}^{2+}, \mathrm{Fe}^{2+}, \mathrm{Cd}^{2+}$ and $\mathrm{Hg}^{2+}$. This parameter indicates a negative significance with $\mathrm{DO}, \mathrm{SO}_{3}^{2-}, \mathrm{Na}^{+}, \mathrm{K}^{+}, \mathrm{NO}_{2}^{-}, \mathrm{NO}_{3}^{-}$and $\mathrm{Pb}^{2+}$ (Table 4).

The SS level of Tuzakl1 Pond is between 2.36 and $10.78 \mathrm{mg} \mathrm{L}^{-1}$. The lowest level of suspended solids was observed at S1 in January and the highest at S2 in September. This parameter at $\mathrm{P}<0.01$ and $\mathrm{P}<0.05$ significance levels exhibits a high positive significance $(\mathrm{r} \geq 0.7)$ relationship with salinity, WT, EC, $\mathrm{BOD}_{5}, \mathrm{TH}$, $\mathrm{TA}, \mathrm{Mg}^{2+}, \mathrm{Cd}^{2+}$ and $\mathrm{Hg}^{2+}$. This parameter indicates a negative significance relation with $\mathrm{DO}, \mathrm{SO}_{3}{ }^{2-}, \mathrm{Na}^{+}, \mathrm{K}^{+}$, $\mathrm{NO}_{2}^{-}, \mathrm{NO}_{3}^{-}$and $\mathrm{Pb}^{2+}$ (Table 4).

The COD value of this pond varied between 1.00 and $6.26 \mathrm{mg} \mathrm{L}^{-1}$; the lowest value was observed at $\mathrm{S} 1$ in December, and the highest was observed at S2 and S3 in June. According to the inland water, quality criteria of the WHO and SWQMR, the COD value of this pond is Class I ( $\leq 25 \mathrm{mg} \mathrm{L}^{-1}$ ) (Table 3) [23-24]. This parameter at $\mathrm{P}<0.01$ and $\mathrm{P}<0.05$ significance levels exhibits a high positive significance $(\mathrm{r} \geq 0.7)$ relationship with $\mathrm{BOD}_{5}$, $\mathrm{SO}_{4}^{2-}, \mathrm{TA}, \mathrm{Ca}^{2+}, \mathrm{Cu}^{2+}, \mathrm{Zn}^{2+}$ and a negative significance relationship with $\mathrm{DO}, \mathrm{PO}_{4}{ }^{3-}, \mathrm{NO}_{2}^{-}$and $\mathrm{NH}_{4}^{+}$(Table 4).

The values of biological oxygen demand varied between 0.62 and $2.20 \mathrm{mg} \mathrm{L}^{-1}$ in the pond. The lowest value was observed at S3 in March and the highest at $\mathrm{S} 1$ in June. According to the water quality classification regulations, the $\mathrm{BOD}_{5}$ values of the pond fit Class I $\left(<4 \mathrm{mg} \mathrm{L}^{-1}\right)$ (Table 3) [23-24]. The $\mathrm{BOD}_{5}$ at $\mathrm{P}<0.01$ and $\mathrm{P}<0.05$ significance levels exhibits a high positive significance $(\mathrm{r} \geq 0.7)$ relationship with $\mathrm{WT}, \mathrm{EC}$, $\mathrm{SS}, \mathrm{COD}, \mathrm{Cl}^{-}, \mathrm{TA}, \mathrm{Ca}^{2+}, \mathrm{Mg}^{2+}, \mathrm{Cu}^{2+}$ and a negative significance relation with $\mathrm{DO}$ and $\mathrm{NO}_{2}^{-}$(Table 4).

The chlorine value of the pond varied between 3.36 and $6.72 \mathrm{mg} \mathrm{L}^{-1}$. The lowest $\mathrm{Cl}^{-}$concentration was observed at S3 in February, whereas the highest concentration was observed at $\mathrm{S} 1$ in December. According to the water quality criteria, the chlorine value is Class $\mathrm{I}\left(<10 \mathrm{mg} \mathrm{L}^{-1}\right)$ (Table 3) [23-24]. This parameter at $\mathrm{P}<0.01$ and $\mathrm{P}<0.05$ significance levels exhibits a high positive significance ( $\mathrm{r} \geq 0.7$ ) relationship with salinity, $\mathrm{EC}, \mathrm{BOD}_{5}, \mathrm{PO}_{4}^{3-}, \mathrm{Mg}^{2+}$ and $\mathrm{Ni}^{2+}$, and a negative significance relation with $\mathrm{DO}, \mathrm{SO}_{3}{ }^{2-}$ and $\mathrm{NO}_{2}$ (Table 4).

The phosphorus level of this pond varied between 0.0009 and $0.5880 \mathrm{mg} \mathrm{L}^{-1}$; the lowest concentration was observed at S3 in January and the highest at S1 in November. Phosphate at $\mathrm{P}<0.01$ and $\mathrm{P}<0.05$ significance levels exhibits a positive significance $(\mathrm{r} \geq 0.5)$ relationship with $\mathrm{EC}, \mathrm{Cl}^{-}, \mathrm{Mg}^{2+} \mathrm{NH}_{4}^{+}, \mathrm{Ni}^{2+}$, and a negative 
significance relation with $\mathrm{DO}, \mathrm{COD}, \mathrm{SO}_{3}{ }^{2-}, \mathrm{SO}_{4}{ }^{2-}, \mathrm{Na}^{+}$, $\mathrm{K}^{+}, \mathrm{NO}_{2}^{-}$and $\mathrm{Pb}^{2+}$ (Table 4). According to SWQMR, the pond can be classified as Class II $\left(<0.65 \mathrm{mg} \mathrm{L}^{-1}\right)$ in terms of phosphate (Table 3) [23-24]. The maximum phosphate concentration of the pond is observed to be higher than that of Terzi and Küçüksu ponds and lower than that of Bektaş Pond [35-37].

The sulphate concentration of this pond varied between 48.78 and $82.26 \mathrm{mg} \mathrm{L}^{-1}$. The lowest sulphate concentration was detected at $\mathrm{S} 1$ in December while the highest levels were detected at S1 and S2 in July. This parameter at $\mathrm{P}<0.01$ and $\mathrm{P}<0.05$ significance levels exhibits a high positive $(r \geq 0.7)$ relationship with COD, TA and a negative significance relationship with $\mathrm{PO}_{4}^{3-}$, $\mathrm{NO}_{2}^{-}, \mathrm{NH}_{4}^{+}, \mathrm{Fe}^{2+}, \mathrm{Hg}^{2+}$ and $\mathrm{Ni}^{2+}$ (Table 4).

The sulphite concentration varied between 0.64 and $2.06 \mathrm{mg} \mathrm{L}^{-1}$. The lowest concentration of this parameter was observed at S1 in December, whereas the highest level was observed at S2 in April. In terms of sulphite, it is Class II ( $>2 \mathrm{mg} \mathrm{L}^{-1}$ ) (Table 3) [23-24]. Sulphite at $\mathrm{P}<0.01$ and $\mathrm{P}<0.05$ significance levels exhibits a positive $(\mathrm{r} \geq 0.7)$ relationship with $\mathrm{Na}^{+}$and a negative significance relationship with salinity, $\mathrm{EC}, \mathrm{SS}, \mathrm{PO}_{4}^{3-}, \mathrm{Cl}^{-}, \mathrm{NH}_{4}^{+}, \mathrm{Fe}^{2+}$, $\mathrm{Hg}^{2+}$ and $\mathrm{Ni}^{2+}$ (Table 4). The highest amount of sulphite in this pond is higher than that observed in the Eglence and Alpsari ponds [38-39].

The sodium concentration in this pond varied between 38.62 and $76.10 \mathrm{mg} \mathrm{L}^{-1}$. The lowest sodium concentration was observed at $\mathrm{S} 1$ in October, whereas the highest level was observed at $\mathrm{S} 2$ in June. This parameter at $\mathrm{P}<0.01$ and $\mathrm{P}<0.05$ significance levels exhibits a high positive $(\mathrm{r} \geq 0.7)$ relationship with $\mathrm{DO}, \mathrm{SO}_{3}{ }^{2-}, \mathrm{K}^{+}, \mathrm{Mg}^{2+}, \mathrm{NO}_{3}^{-}, \mathrm{Pb}^{2+}, \mathrm{Cu}^{2+}$ and $\mathrm{Zn}^{2+}$, and a negative significance relationship with salinity, WT, EC, $\mathrm{SS}, \mathrm{PO}_{4}^{3-}, \mathrm{NH}_{4}^{+}, \mathrm{Ni}^{2+}, \mathrm{CD}^{2+}, \mathrm{Hg}^{2+}$ and $\mathrm{Fe}^{2+}$ (Table 4).

In this study, the potassium concentration varied between 4.74 and $17.20 \mathrm{mg} \mathrm{L}^{-1}$. The lowest potassium concentration was detected at S1 in October and the highest at $\mathrm{S} 2$ in June. Potassium exhibits a high positive $(\mathrm{r} \geq 0.7)$ relationship with $\mathrm{DO}, \mathrm{Na}^{+}, \mathrm{NO}^{3-}, \mathrm{Pb}^{2+}$ and a negative significance relationship with salinity, $\mathrm{pH}, \mathrm{WT}$, $\mathrm{EC}, \mathrm{SS}, \mathrm{Cl}^{-}, \mathrm{PO}_{4}^{3-}, \mathrm{Mg}^{2+}, \mathrm{NH}_{4}^{+}, \mathrm{Fe}^{2+}, \mathrm{Cd}^{2+}, \mathrm{Hg}^{2+}$ and $\mathrm{Ni}^{2+}$ (Table 4).

The TH value of Tuzakl1 Pond varied between 239.74 and $397.00 \mathrm{CaCO}_{3} \mathrm{mg} \mathrm{L}^{-1}$. The lowest $\mathrm{TH}$ was observed at S3 in December while the highest was observed at S3 in August. This parameter exhibits a high positive ( $r \geq 0.7$ ) relationship at $\mathrm{P}<0.01$ and $\mathrm{P}<0.05$ significance levels, with TA and $\mathrm{SS}$ and a negative significance relationship with $\mathrm{DO}, \mathrm{NO}_{2}^{-}, \mathrm{NH}_{4}^{+}, \mathrm{Pb}^{2+}$ (Table 4).

The total alkalinity (TA) of this study varied between 242.96 and $305.5 \mathrm{CaCO}_{3} \mathrm{mg} \mathrm{L}^{-1}$. The lowest TA concentration was observed at $\mathrm{S} 3$ in December, whereas the highest was observed at S2 in September. This parameter at $\mathrm{P}<0.01$ and $\mathrm{P}<0.05$ significance levels exhibits a high positive $(\mathrm{r} \geq 0.7)$ relationship with $\mathrm{WT}$, $\mathrm{EC}, \mathrm{SS}, \mathrm{COD}, \mathrm{BOD}_{5}, \mathrm{TH}, \mathrm{SO}_{4}{ }^{2-}, \mathrm{Mg}^{2+}$ and $\mathrm{Cd}^{2+}$, and a negative significance relationship with $\mathrm{DO}, \mathrm{NO}_{2}^{-} \mathrm{NH}_{4}^{+}$ and $\mathrm{Pb}^{2+}$ (Table 4).
The magnesium concentration of this pond varied between 22.20 and $55.28 \mathrm{mg} \mathrm{L}^{-1}$; the lowest concentration was observed at S2 and S3 in February, whereas the highest concentration was observed at S2 in July. Magnesium exhibits a high positive $(\mathrm{r} \geq 0.7)$ relationship with salinity, WT, EC, SS, $\mathrm{BOD}_{5}, \mathrm{Cl}^{-}$, $\mathrm{PO}_{4}^{3-}$ and TA, and a negative significance relationship with $\mathrm{DO}, \mathrm{SO}_{3}{ }^{2-}, \mathrm{K}^{+}, \mathrm{NO}_{2}^{-}$and $\mathrm{Pb}^{2+}$ at $\mathrm{P}<0.01$ and $\mathrm{P}<0.05$ significance levels (Table 4).

The calcium concentration was observed to be between 22.82 and $80.58 \mathrm{mg} \mathrm{L}^{-1}$. The lowest calcium concentration was observed at S1 in February while the highest concentration was observed at S2 in July. According to Pearson's correlation, it exhibits a high positive $(\mathrm{r} \geq 0.7)$ relationship at $\mathrm{P}<0.01$ and $\mathrm{P}<0.05$ significance levels with $\mathrm{COD}, \mathrm{BOD}_{5}, \mathrm{Na}^{+}, \mathrm{NO}_{3}^{-}, \mathrm{Cu}^{2+}$ and $\mathrm{Zn}^{2+}$, and a negative significance relationship with $\mathrm{NO}_{2}^{-}$(Table 4).

The nitrite concentration of Tuzakl1 varied between 0.0001 and $0.0151 \mathrm{mg} \mathrm{L}^{-1}$. The lowest concentration of nitrite was observed at all the stations and in all the months except April, June and September, while the highest concentration was observed at S2 in February. The nitrite level of this pond, according to the criteria, is Class II $\left(<0.06 \mathrm{mg} \mathrm{L}^{-1}\right)$ (Table 3). [23-24]. This parameter at $\mathrm{P}<0.01$ and $\mathrm{P}<0.05$ significance levels exhibits a high positive $(\mathrm{r} \geq 0.7)$ relationship with $\mathrm{DO}, \mathrm{COD}, \mathrm{BOD}_{5}, \mathrm{SO}_{3}{ }^{2-}, \mathrm{K}^{+}$and $\mathrm{CD}^{2+}$, and a negative significance relationship with all the parameters except these (Table 4). The nitrite level of Tuzaklı was observed to be higher than that of Karagöl and Maruf ponds [40-41].

The nitrate concentration of this pond varied between 1.64 and $13.10 \mathrm{mg} \mathrm{L}^{-1}$. The lowest concentration of nitrate was observed at S3 in January, whereas the highest level was observed at S2 in June. This pond can be classified as Class II in terms of nitrate $\left(>10 \mathrm{mg} \mathrm{L}^{-1}\right.$ ) (Table 3) [23-24]. This parameter at $\mathrm{P}<0.01$ and $\mathrm{P}<0.05$ significance levels exhibits a high positive $(r \geq 0.7)$ relationship with $\mathrm{K}^{+}, \mathrm{Ca}^{2+}, \mathrm{Pb}^{2+}$, $\mathrm{Cu}^{2+}$ and $\mathrm{Zn}^{2+}$, and a negative significance relationship with salinity, $\mathrm{pH}, \mathrm{EC}, \mathrm{SS}, \mathrm{NO}_{2}^{-}, \mathrm{Fe}^{2+}, \mathrm{Cd}^{2+}$ and $\mathrm{Hg}^{2+}$ (Table 4). We observed that the nitrate value of this pond was lower than that of Küçüksu Pond, but higher than that of the Ulugöl Lake [36, 42].

The ammonium concentration varied between

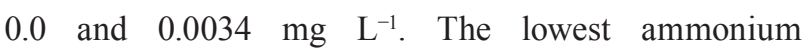
concentration was observed at all the stations during January, February and March, whereas the highest level was detected at S2 in November. According to the water quality classification criteria, Tuzaklı Pond is Class I $\left(<0.2 \mathrm{mg} \mathrm{L}^{-1}\right)$ (Table 3) [23-24]. This parameter exhibits a high positive relationship at $\mathrm{P}<0.01$ and $\mathrm{P}<0.05$ significance levels with $\mathrm{PO}_{4}^{3-}$, and a negative significance relationship with $\mathrm{DO}, \mathrm{pH}, \mathrm{COD}, \mathrm{SO}_{4}{ }^{2-}$, $\mathrm{SO}_{3}{ }^{2-}, \mathrm{Na}^{+}, \mathrm{K}^{+}, \mathrm{TH}, \mathrm{TA}, \mathrm{NO}_{2}^{-}, \mathrm{Cd}^{2+}$ and $\mathrm{Pb}^{2+}$ (Table 4).

The iron level of Tuzakl1 Pond was observed to be between 0.00 and $0.0070 \mu \mathrm{g} \mathrm{L} \mathrm{L}^{-1}$. The lowest concentration of iron was observed in September at 
all the stations, while the highest was detected at S2 in October. According to SWQMR's surface water quality criteria, this pond was Class I in terms of iron $(\leq 300 \mu \mathrm{g}$ $\mathrm{L}^{-1}$ ) (Table 3) [23-24]. This parameter at $\mathrm{P}<0.01$ and $\mathrm{P}$ $<0.05$ significance levels exhibit a high positive $(\mathrm{r} \geq 0.5)$ significance relationship with salinity, WT, EC, $\mathrm{Hg}^{2+}$ and $\mathrm{Ni}^{2+}$, and a negative significance relationship with $\mathrm{DO}, \mathrm{SO}_{3}{ }^{2-} \mathrm{SO}_{4}{ }^{2-}, \mathrm{Na}^{+}, \mathrm{K}^{+}, \mathrm{NO}_{2}{ }^{-}, \mathrm{NO}_{3}{ }^{-}$and $\mathrm{Pb}^{2+}$ (Table 4).

The lead level of this pond has been identified to be between 0.30 and $2.10 \mu \mathrm{g} \mathrm{L}^{-1}$; the lowest concentration was observed at all the stations in September, and the highest concentration was observed at S2 in June. This parameter at $\mathrm{P}<0.01$ and $\mathrm{P}<0.05$ significance levels exhibit a high positive $(r \geq 0.7)$ significance relationship with $\mathrm{DO}, \mathrm{Na}^{+}, \mathrm{K}^{+}, \mathrm{NO}_{3}^{-}$and $\mathrm{Cu}^{2+}$, and a negative significance relationship with salinity, $\mathrm{pH}, \mathrm{WT}, \mathrm{EC}, \mathrm{SS}$, $\mathrm{PO}_{4}^{3-}, \mathrm{TH}, \mathrm{Mg}^{2+}, \mathrm{NO}_{2}^{-}, \mathrm{NH}_{4}^{+}, \mathrm{Fe}^{2+}, \mathrm{Cd}^{2+}, \mathrm{Hg}^{2+}$ and $\mathrm{Ni}^{2+}$ (Table 4). According to SWQMR's criteria for surface water quality, in terms of lead level, Tuzaklı Pond has been classified as Class I $\left(\leq 10 \mu \mathrm{g} \mathrm{L}^{-1}\right)$ (Table 3) [23-24].

The copper level of this pond varied between 0.0 and $14.00 \mu \mathrm{g} \mathrm{L}^{-1}$. The lowest copper value was observed at all stations in January, February and March, and the highest level was observed at S1 in June. This element at $\mathrm{P}<0.01$ and $\mathrm{P}<0.05$ significance levels exhibits a high positive $(r \geq 0.7)$ significance relationship with $\mathrm{COD}$, $\mathrm{BOD}_{5}, \mathrm{Ca}^{2+}, \mathrm{Na}^{+}, \mathrm{NO}_{3}^{-}, \mathrm{Pb}^{2+}$ and $\mathrm{Zn}^{2+}$, and a negative significance relationship with $\mathrm{NO}_{2}^{-}$and $\mathrm{Hg}^{2+}$ (Table 4). According to SWQMR's surface water quality criteria, in terms of copper level this pond has been classified as Class I, which indicates clean water $\left(\leq 20 \mu \mathrm{g} \mathrm{L}^{-1}\right)$ (Table 3) [23-24].

The cadmium level of Tuzaklı Pond varied between 0.00 and $0.50 \mu \mathrm{g} \mathrm{L}^{-1}$. The lowest cadmium level was observed at all the stations in January, whereas the highest level was identified at S2 and S3 in September. This metal exhibits a positive $(r \geq 0.7)$ relationship at $\mathrm{P}<0.01$ and $\mathrm{P}<0.05$ significance levels with salinity, WT, EC, SS, TH and TA and a negative significance relationship with $\mathrm{DO}, \mathrm{Na}^{+}, \mathrm{K}^{+}, \mathrm{NO}_{3}^{-}, \mathrm{NH}_{4}^{+}$and $\mathrm{Pb}^{2+}$ (Table 4). According to the criteria of water classes, in terms of cadmium, Tuzaklı has been identified as Class I $\left(\leq 2 \mu \mathrm{g} \mathrm{L}^{-1}\right)$ (Table 3$)$ [23-24].

The mercury level varied between 0.0 and $0.0070 \mu \mathrm{g} \mathrm{L}^{-1}$; the lowest level was observed at all the stations in January, February, March, April, May, June and July, whereas the highest level was detected at S2 in October. This element exhibits a high positive $(r \geq 0.7)$ relationship at $\mathrm{P}<0.01$ and $\mathrm{P}<0.05$ significance levels with salinity, EC and $\mathrm{SS}$, and a negative significance relationship with $\mathrm{DO}, \mathrm{SO}_{3}{ }^{2-}, \mathrm{SO}_{4}{ }^{2-}, \mathrm{Na}^{2+}, \mathrm{K}^{+}, \mathrm{NO}_{2}^{-}, \mathrm{NO}_{3}^{-}$, $\mathrm{Cu}^{2+}$ and $\mathrm{Pb}^{2+}$ (Table 4). According to the water quality classification criteria, the mercury level of this study can be classified as Class I $\left(\leq 0.1 \mu \mathrm{g} \mathrm{L}^{-1}\right)$ (Table 3) [23-24].

In this study, the nickel level varied between 3.00 and $13.0 \mu \mathrm{g} \mathrm{L}^{-1}$. The lowest level of nickel was observed at all the stations in February and April, and the highest level at S2 and S3 in November. This parameter exhibits a high positive ( $\mathrm{r} \geq 0.7$ ) relationship at $\mathrm{P}<0.01$ and $\mathrm{P}<0.05$ significance levels with $\mathrm{Cl}^{-}, \mathrm{PO}_{4}^{3-}$ and $\mathrm{Fe}^{2+}$, and a negative significance relationship with $\mathrm{DO}, \mathrm{SO}_{3}{ }^{2-}, \mathrm{SO}_{4}{ }^{2-}$, $\mathrm{Na}^{2+}, \mathrm{K}^{+}$and $\mathrm{Pb}^{2+}$ (Table 4). According to the water quality classification criteria, the nickel level of Tuzakl1 is Class I $\left(\leq 20 \mu \mathrm{g} \mathrm{L}^{-1}\right)$ (Table 3) [23-24].

Tuzakl1 Pond's zinc level varied between 1.0 and $25.0 \mu \mathrm{g} \mathrm{L}^{-1}$. The lowest level of zinc was observed at $\mathrm{S} 1$ in January, whereas the highest level was at S2 in June. In this study, the zinc value at $\mathrm{P}<0.01$ and $\mathrm{P}<0.05$ significance levels exhibit a positive significance $(\mathrm{r} \geq 0.7)$ relationship with $\mathrm{COD}, \mathrm{BOD}_{5}, \mathrm{Na}^{+}, \mathrm{Ca}^{2+}, \mathrm{NH}_{4}^{+}$ and $\mathrm{Cu}^{2+}$, and a negative significance relationship with $\mathrm{NO}_{2}^{-}$and $\mathrm{Hg}^{2+}$ (Table 4). According to the water quality classification criteria, the zinc level of this pond is Class I $\left(\leq 200 \mu \mathrm{g} \mathrm{L}^{-1}\right)$ (Table 3) [23-24].

From the results of univariate statistics, it is possible to deduce definite features or conclusions for the examined data even though the results would be unilateral; however, multivariate statistical methods such as PCA and HCA are applied to multi-dimensional datasets because they do not require a lot of time. Statistical analyses were performed on the results related to the 28 parameters that were obtained from the 36 water samples that were obtained on a monthly basis from the three stations in Tuzaklı Pond based on the mean values of various stations and seasons.

HCA was used to detect the spatial similarity between the stations. Based on the stations, the parameters were approximately divided into two main groups: clusters A and B (Fig. 2). Cluster A comprised stations $\mathrm{S} 1$ and $\mathrm{S} 3$ while cluster B comprised station S2. When an internal analysis of cluster A was conducted, it could be observed that S1 and S3 exhibited similar properties in terms of pollutant load among themselves and, therefore, may be similar in terms of the pollutant source.

In cluster $\mathrm{B}, \mathrm{S} 2$ is different from other stations because it is located at a station where Gavur Creek flows into the pond as the water source that feeds the pond; however, this factor is not statistically significant (Fig. 2). According to the results of this analysis, it can be concluded that Tuzaklı Pond exhibits two different water qualities based on the stations.

Based on the temporal HCA results, clusters can be approximately divided into two main groups in terms of seasons: clusters A and B (Fig. 3). Cluster A depicts the winter and spring seasons, whereas cluster B depicts

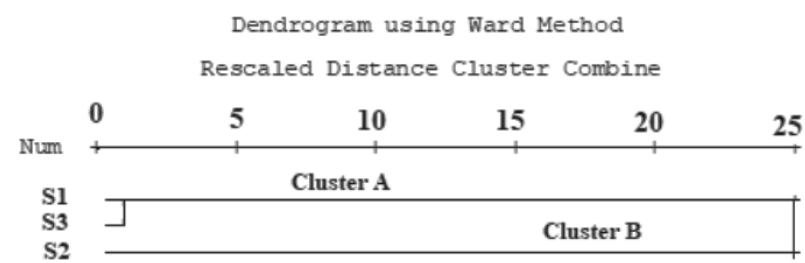

Fig. 2. Dendogram (obtained using the Ward method) showing clusters of variables $(\mathrm{St} .=\mathrm{Site})$. 
Dendrogram using Ward Method

Rescaled Distance Cluster Combine

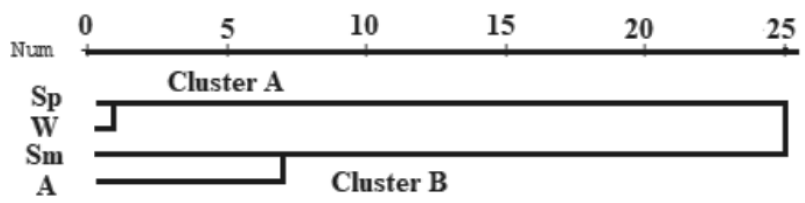

Fig. 3. Dendogram showing clusters of season variables.

Table 5. Varimax rotated factor matrix for the whole data set.

\begin{tabular}{|c|c|c|c|c|}
\hline Variable & PC 1 & PC 2 & PC 3 & PC 4 \\
\hline Eigenvalues & 10.528 & 8.308 & 4.770 & 1.120 \\
\hline Variance $(\%)$ & 37.600 & 29.671 & 17.037 & 4.001 \\
\hline \multirow[t]{2}{*}{ Cumulative (\%) } & 37.600 & 67.270 & 84.307 & 88.308 \\
\hline & \multicolumn{4}{|c|}{ Factor loadings (varimax normalised) } \\
\hline SS & 0.965 & -0.075 & 0.159 & 0.065 \\
\hline $\mathrm{EC}$ & 0.936 & -0.107 & 0.292 & 0.017 \\
\hline WT & 0.931 & 0.111 & 0.207 & 0.068 \\
\hline $\mathrm{Cd}^{2+}$ & 0.929 & -0.053 & -0.106 & -0.141 \\
\hline Salinity & 0.901 & -0.062 & 0.345 & 0.028 \\
\hline TA & 0.863 & 0.400 & -0.193 & 0.090 \\
\hline $\mathrm{Mg}^{2+}$ & 0.813 & 0.199 & 0.389 & 0.181 \\
\hline $\mathrm{TH}$ & 0.793 & 0.138 & -0.224 & 0.107 \\
\hline BOD & 0.762 & 0.591 & 0.174 & 0.041 \\
\hline $\mathrm{pH}$ & 0.741 & 0.189 & 0.049 & 0.049 \\
\hline $\mathrm{Hg}^{2+}$ & 0.703 & -0.399 & 0.332 & -0.024 \\
\hline $\mathrm{Fe}^{2+}$ & 0.656 & -0.084 & 0.571 & -0.067 \\
\hline DO & -0.653 & 0.613 & -0.360 & 0.047 \\
\hline $\mathrm{NO}_{3}^{-}$ & 0.026 & 0.966 & 0.054 & 0.085 \\
\hline $\mathrm{Na}^{+}$ & -0.092 & 0.943 & -0.214 & 0.073 \\
\hline $\mathrm{Cu}^{2+}$ & 0.234 & 0.925 & 0.256 & 0.019 \\
\hline $\mathrm{Pb}^{2+}$ & -0.350 & 0.885 & 0.000 & -0.017 \\
\hline $\mathrm{Zn}^{2+}$ & 0.198 & 0.885 & 0.344 & 0.014 \\
\hline $\mathrm{K}^{+}$ & -0.157 & 0.864 & -0.277 & -0.128 \\
\hline $\mathrm{Ca}^{2+}$ & 0.435 & 0.851 & 0.145 & 0.119 \\
\hline COD & 0.590 & 0.746 & -0.122 & -0.027 \\
\hline $\mathrm{SO}_{3}^{2-}$ & 0.090 & 0.627 & -0.601 & 0.049 \\
\hline $\mathrm{SO}_{4}{ }^{2-}$ & 0.467 & 0.588 & -0.522 & 0.028 \\
\hline $\mathrm{PO}_{4}^{3-}$ & 0.301 & -0.054 & 0.914 & 0.103 \\
\hline $\mathrm{Ni}^{2+}$ & 0.405 & 0.125 & 0.857 & -0.091 \\
\hline $\mathrm{NH}_{4}^{+}$ & -0.091 & 0.021 & 0.843 & 0.100 \\
\hline $\mathrm{Cl}^{-}$ & 0.611 & 0.308 & 0.619 & 0.187 \\
\hline $\mathrm{NO}_{2}^{-}$ & -0.114 & -0.050 & -0.092 & -0.957 \\
\hline
\end{tabular}

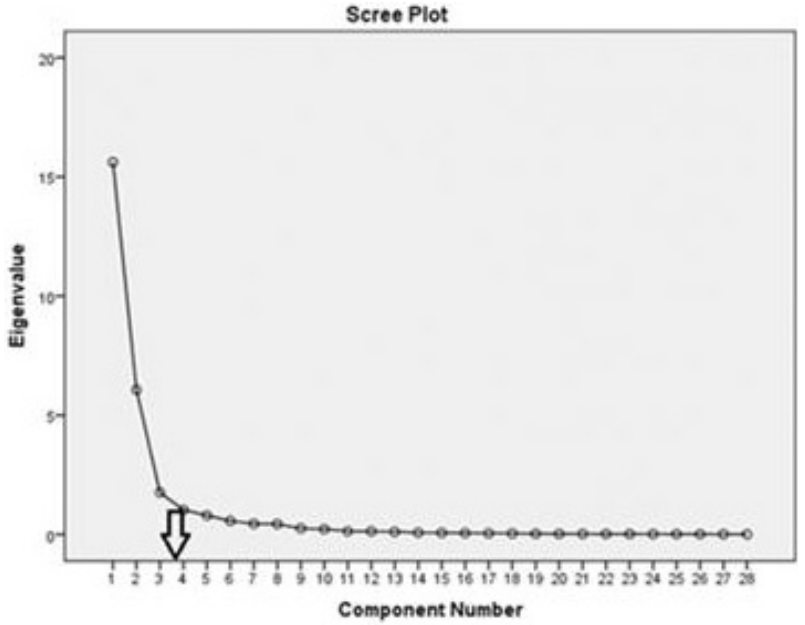

Fig. 4. Component plot.

the summer and autumn seasons. When an internal analysis of cluster A was conducted, the spring and winter seasons were observed to exhibit similarities. In a similar manner, the summer and autumn seasons that constituted cluster B exhibited more similar properties as compared to those exhibited by cluster A.

Before applying PCA, the suitability of PCA was verified by applying the $\mathrm{KMO}$ and Bartlett tests to datasets. While selecting the number of main components, it was supported by incorporating the main components prior to a definite breakage of the Scree plot (Fig. 4) [43-45]. We concluded that four main components can represent the data of Tuzaklı Pond (Table 5).

These main components were obtained with eigenvalues that summarised $88.308 \%$ of the total variance in the dataset (Table 5). The first major component that explains $37.60 \%$ of the total variance is strong positively loaded with SS, EC, water temperature,

\section{Component Plot in Rotated Space}

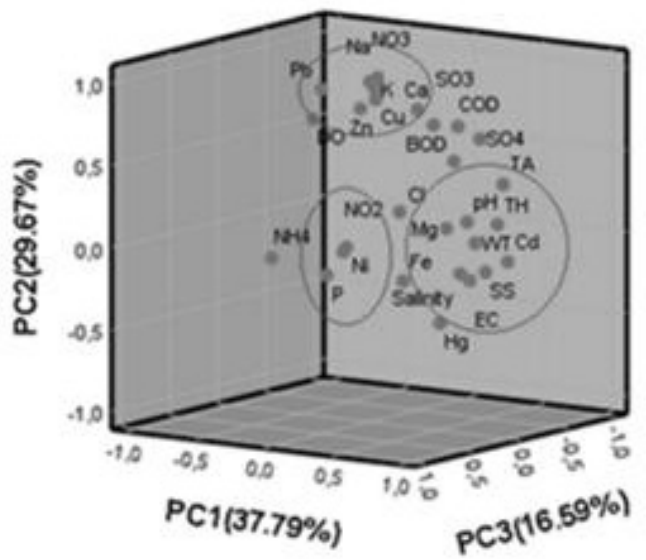

Fig. 5. Scree plot for the principal component model of the monitoring data. 
$\mathrm{Cd}^{2+}$, salinity, $\mathrm{TA}, \mathrm{Mg}^{2+}$, $\mathrm{TH}$ and $\mathrm{BOD}_{5}$; moderately positively loaded with $\mathrm{pH}, \mathrm{Hg}^{2+}, \mathrm{Fe}^{2+}$ and $\mathrm{COD}$; and moderately negatively loaded with dissolved oxygen (Table 5 and Fig. 5). Surface runoff and erosion of the rocks that occur because of precipitation, which is one of the effects of climate factors, can be a source of this component. In addition, it may cause the formation and dissolution of soluble salts (natural), such as limestone and gypsum dissolution, which constitute the soil structure, anions and cations that give hardness to water, with the effect of erosion after rain [46-47]. This main component is characterized by the physical parameters and soluble salts.

The second major component that constitutes $29.67 \%$ of the total variance exhibits strong positive loading with $\mathrm{NO}_{3}^{-}, \mathrm{Na}^{+}, \mathrm{Cu}^{2+}, \mathrm{Pb}^{2+}, \mathrm{Zn}^{2+}, \mathrm{K}^{+}$and $\mathrm{Ca}^{2+}$ (Table 5 and Fig. 5). This factor represents the heavy metals and cations [47].

The third PC (principal component), which accounts for $17.03 \%$ of the total variance, exhibits high positive loading with $\mathrm{PO}_{4}^{3-}, \mathrm{Ni}^{2+}$ and $\mathrm{NH}_{4}^{+}$(Table 5 and Fig. 5). This component represents the nutrient elements (phosphate and ammonium).

The fourth PC has a minimum deviation of $4.0 \%$. $\mathrm{NO}_{2}^{-}$exhibits a strong negative loading (Table 5 and Fig. 5). This component may be formed due to the increased drainage observed in the field after rainfall because of agricultural activities that have been conducted by farmers using nitrogen fertilizers around the pond and because of the natural weather conditions [48-49].

\section{Conclusions}

Various multivariate statistical techniques have been successfully used to determine the temporal and spatial variations in the surface water quality of the pond, the main pollutants and the sources of the main pollutants in the study area.

The majority of components in Tuzaklı Pond, in terms of water quality classes, represent Class I, while water temperature, $\mathrm{PO}_{4}^{3-}, \mathrm{SO}_{3}{ }^{2-}, \mathrm{NO}_{2}^{-}$and $\mathrm{NO}_{3}{ }^{-}$represent Class II. Thus, the pond is observed to generally contain less-polluted water. Pond water has the potential to be used as drinking water and for irrigation purposes; it can also be used for fish breeding (excluding trout) and for recreational purposes. Aquatic life in Tuzakl1 is threatened because of the excessive load of the nutrient elements. To improve the water quality, it is necessary to ensure the controlled usage of chemical fertilizers, which are extensively used in agricultural areas, and to prevent the animal wastes from reaching the pond.

The water of the pond is not a threat in terms of heavy metal load. It can be temporally concluded using HCA that the summer and autumn seasons exhibit more similar characteristics than that exhibited by the remaining seasons. According to the HCA result, when it is spatially assessed, two different water qualities were observed in the pond; thus it can be concluded that the station, which was located at the water source, made the difference. PCA depicted that the four major components accounted for $88.308 \%$ of the total variation. These major components generally reveal most of the changes in water quality as physical parameters, soluble salts (natural) and ammonium and phosphorus (agricultural activity) from the nutrient elements. Tuzakl1 Pond poses a threat to aquatic life, especially due to the excessive ratio of the nutrient elements. To improve water quality of the pond, it is necessary to ensure controlled usage of chemical fertilizers that are extensively used in agricultural areas and to prevent animal wastes from reaching the pond.

Successful implementation of HCA and PCA analyses allows us to interpret the complex datasets of water quality, recognise any temporal or spatial variation in water quality, and identify the sources or factors of latent pollution. Integrating these methods in the existing water quality enhancement activities will allow managers to achieve both time-based and financial benefits in case of water monitoring plans in order to identify the pollution sources in different regions and to set priorities for improving water quality.

\section{References}

1. EGEMEN Ö., SUNLU U. Water Quality. E.Ü. Fisheries Faculty. Publication No. 14, Bornova-İzmir. IV. Edition, 153, 2003

2. CAUSAPE J., QUILEZ D., ARAGUES R. Assessment of irrigation and environmental quality at the hydrological basin level I. Irrigation quality. Agricultural Water Management, 70, 195, 2004.

3. IQBAL M., ABBAS M., ARSHAD M., HUSSAIN T., KHAN A. U., MASOOD N., TAHIR M. A., HUSSAIN S.M., BOKHARI T.H., KHERA R. A. Gamma radiation treatment for reducing cytotoxicity and mutagenicity in industrial wastewater. Polish Journal of Environmental Studies, 24 (6), 2745, 2015.

4. IQBAL M. Vicia faba bioassay for environmental toxicity monitoring: A review. Chemosphere, 144, 785, 2016.

5. IQBAL M., ABBAS M., NISAR J., NAZIR A. Bioassays based on higher plants as excellent dosimeters for ecotoxicity monitoring: A review. Chemestry International, 5 (1), 1, 2019.

6. TAŞ B. Investigation of Water Quality of Derbent Dam Lake (Samsun). Ekoloji, 15 (61), 6, 2006.

7. MOSS B. Ecology of Freshwaters, Man and Medium. 2nd ed. Blackwell Sci. Pub. Oxford.Popovski, 1988.

8. WEHR J.D., SHEATH R.G., Freshwater Algae of North America. Ecology and Classification. Aquatic Ecology Series. Academic Press. 918, 2003.

9. VEGA M., PARDO R., BARRADO E., DEBAN L. Assessment of seasonal and polluting effects on the quality of river water by exploratory data analysis. Water Research, 32 (12), 3581, 1998.

10. SINGH K.P., MALIK A., MOHAN D. AND SINHA S. Multivariate statistical techniques for the evaluation of spatial and temporal variations in water quality of Gomti River (India) - a case study, Water Res., 38, 3980, 2004. 
11. SHRESTHA S., KAZAMA F. Assessment of surface water quality using multivariate statistical techniques: A case study of the Fuji River Basin, Japan. Environmental Modelling and Software, 22 (4), 464, 2007.

12. DALAL S.G., SHIRODKAR P.V., JAGTAP T.G., NAIK B.G., RAO G.S. Evaluation of significant sources influencing the variation of water quality of Kandla creek, Gulf of Katchchh, using PCA. Environmental Monitoring and Assessment, 163 (1-4), 49, 2010.

13. REGHUNATH R., MURTHY T.R.S., RAGHAVAN B.R. The utility of multivariate statistical techniques in hydrogeochemical studies: an example from Karnataka, India. Water Research, 36, 2437, 2002.

14. SIMEONOV V., STRATIS J.A., SAMARA C., ZACHARIADIS G., VOUTSA D., ANTHEMIDIS A., SOFONIOU M., KOUIMTZIS T. Assessment of the surface water quality in Northern Greece. Water Research, 37 (17), 4119, 2003.

15. KAZI T.G., ARAIN M.B., JAMALI M.K., JALBANI N., AFRIDI H.I., SARFRAZ R.A., BAIG J.A., SHAH A.Q. Assessment of water quality of polluted lake using multivariate statistical techniques: A case study, Ecotox. Environ. Safe. 72, 301, 2009.

16. SINGH K. P., MALIK A., SINHA S. Water quality assessment and apportionment of pollution sources of Gomti river (India) using multivariate statistical techniques-A case study. Analytica Chimica Acta, 538 (12), 355, 2005.

17. LIN W.S., LEE M., HUANG Y.C., DEN W. Identifying water recycling strategy using multivariate statistical analysis for high-tech. Resources, Conservation and Recycling, 94, 35, 2015.

18. HEROJEET R., RISHI M. S., LATA R., DOLMA K. Quality characterization and pollution source identification of surface water using multivariate statistical techniques, Nalagarh Valley, Himachal Pradesh, India. Applied Water Science, 7 (5), 2137, 2017.

19. Climate-Data.org. Available online: https://tr.climate-data. org/location/28797/ (12.08.2018).

20. APHA. Standard methods for the examination of water and wastewater, American Public Health Association. Washington. 21 $1^{\text {st }}$ ed., 1082, 2005.

21. ANONYMOUS. Standard methods for the examination of water and wastewater. American Public Health 486 Association, 7th Edition, Washington, USA, 1998.

22. ŞENGÜL Ü. Comparing determination methods of detection and quantification limits for aflatoxin analysis in hazelnut. Journal of Food and Drug Analysis, 24 (1), 56, 2016.

23. GORCHEV H.G., OZOLINS G. WHO guidelines for drinking-water quality. WHO Chronicle. 38 (3), 104, 2011.

24. SWQMR. Regulation on the surface water quality management. Number of official gazettes: 29797 and 29327, 2016.

25. ALPAR R. Multivariate statistical methods with applications. Detay Publishing, Ankara, 820, 2017.

26. YILMAZ ÖZTÜRK B., AKKÖZ C. Investigation of water quality of Apa Dam Lake (Çumra-Konya) and according to the evolution of PCA. Biological Diversity and Conservation 7 (2), 136, 2014.

27. SHANTHAKUMAR S. Assessment of seasonal variations in surface water quality of Cooum River in Chennai, India - a Statistical Approach, 18 (3), 527, 2016.

28. GE J., RAN G., MIAO W., CAO H., WU S., CHENG L. Water quality assessment of Gufu River in three gorges reservoir (China) using multivariable statistical methods.
Advance Journal of Food Science and Technology, 5(7), 908, 2013.

29. KALAYCI Ş. SPSS applied multivariable statistical techniques. Asil Publication Distribution, 426, 2009.

30. PARINET B., LHOTE A., LEGUBE B. Principal component analysis: an appropriate tool for water quality evaluation and management application to a tropical lake system. Ecol Model, 178, 295, 2004.

31. ÖZDEMIR Ö. Application of multivariate statistical methods for water quality assessment of Karasu Sarmisakli Creeks and Kizilirmak River in Kayseri, Turkey. Polish Journal of Environmental Studies, 25 (3), 1149, 2016.

32. BYRNE P., RUNKEL R.L., WALTON-DAY K. Synoptic sampling and principal components analysis to identify sources of water and metals to an acid mine drainage stream. Environmental Science and Pollution Research, 24 (20), 17220, 2017.

33. HAIR J.F., BLACK W.C., BABIN B.J., ANDERSON R.E. Multivariate data analysis, Prentice Hall, Upper Saddle River, NJ 07458, 116, 2009.

34. LIU C., LIN K., KUO Y. Application of factor analysis in the assessment of groundwater quality in a Blackfoot Disease area in Taiwan. Science of the Total Environment, 313 (1-3), 77, 2003.

35. MUTLU E., AYDIN-UNCUMUSAOGLU A. Analysis of spatial and Temporal water pollution patterns in Terzi Pond by using multivariate statistical methods. Fresenius Environmental Bulletin. 27 (5), 2900, 2018.

36. MUTLU E., AYDIN UNCUMUSAOĞLU A. Investigation of Water Quality of Küçüksu Pond (Taşköprü-Kastamonu). Yunus Research Bulletin, 17 (3), 2017.

37. AYDIN UNCUMUSAOĞLU A. Statistical assessment of water quality parameters for pollution source identification in Bektaş Pond (Sinop, Turkey). Global Nest Journal, 20 (1), 151, 2018.

38. AYDIN UNCUMUSAOĞLU A., MUTLU E. Determination of water quality and usability level of Eğlence pond (Boyabat, Sinop). Alınteri Journal of Agricultural Sciences, 32 (2), 25, 2017.

39. MUTLU E., AYDIN UNCUMUSAOĞLU A. Investigation of the Water Quality of Alpsarı Pond (Korgun-Çankırı) 1. Turkish Journal of Fisheries and Aquatic Sciences, 17, 1231, 2017.

40. MUTLU E., YANIK T., DEMIR T. Karagöl (Hafik - Sivas) 'ün Su Kalitesinin İncelenmesi, Alınteri Zirai Bilimler Dergisi, 24 (B), 35, 2013.

41. MUTLU E., KUTLU B. Determining The Water Quality of Maruf Dam (Boyabat - Sinop), 32 (1), 81, 2017.

42. TAŞ B., CANDAN AY., CAN Ö., TOPKARA S. Some physico-chemical properties of Ulugöl (Ordu). Journal of Fisheries Sciences.com, 4 (3), 254, 2010.

43. AYDIN UNCUMUSAOĞLU A., AKKAN T. Assessment of Yağlidere stream water quality using multivariate statistical techniques. Polish Journal of Environmental Studies, 26 (4), 1715, 2017.

44. STANISZEWSKI R., JUSIK S., BOROWIAK K., BYKOWSKI J., HUGH DAWSON F. Temporal and Spatial Variations of Trophic Status of a Small Lowland River, Pol. J. Environ. Stud. 28 (1), 1, 2019.

45. ÖZDEMIR Ö. Application of multivariate statistical methods for water quality assessment of Karasu Sarmisakli Creeks and Kizilirmak River in Kayseri, Turkey. Polish Journal of Environmental Studies, 25 (3), 1149, 2016.

46. CVEJANOV J., ŠKRBIĆ B.D. Application of principal component and hierarchical cluster analyses in the classification of Serbian bottled waters and a comparison 
with waters from some other European countries. Journal of the Serbian Chemical Society, 82 (6), 711, 2017.

47. BILGIN YILDIRIM H. Heavy Metals in Freshwater Ecosystems, Ankara, 2016.

48. KAZAMA F., YONEYAMA M. Nitrogen generation in the Yamanashi prefecture and its effects on the groundwater pollution, International Environmental Science, 15 (4), 293, 2002.
49. AKKAN T., YAZICIOGLU O., YAZICI, R., YILMAZ M. Assessment of irrigation water quality of Turkey using multivariate statistical techniques and water quality index: Sidd1klı Dam Lake. Desalination and Water Treatment 115, 261, 2018. 